\title{
Gas-to-dust mass ratios in local galaxies over a 2 dex metallicity range ${ }^{\star}$
}

\author{
A. Rémy-Ruyer ${ }^{1}$, S. C. Madden ${ }^{1}$, F. Galliano ${ }^{1}$, M. Galametz ${ }^{2}$, T. T. Takeuchi ${ }^{3}$, R. S. Asano ${ }^{3}$, S. Zhukovska ${ }^{4,5}$, \\ V. Lebouteiller ${ }^{1}$, D. Cormier ${ }^{5}$, A. Jones ${ }^{6}$, M. Bocchio ${ }^{6}$, M. Baes ${ }^{7}$, G. J. Bendo ${ }^{8}$, M. Boquien ${ }^{9}$, A. Boselli ${ }^{9}$, \\ I. DeLooze ${ }^{7}$, V. Doublier-Pritchard ${ }^{10}$, T. Hughes ${ }^{7}$, O. Ł. Karczewski ${ }^{11}$, and L. Spinoglio ${ }^{12}$ \\ ${ }^{1}$ Laboratoire AIM, CEA/IRFU/Service d'Astrophysique, Université Paris Diderot, Bat. 709, 91191 Gif-sur-Yvette, France \\ e-mail: aurelie.remy@cea.fr \\ 2 Institute of Astronomy, University of Cambridge, Madingley Road, Cambridge CB3 OHA, UK \\ 3 Division of Particle and Astrophysical Science, Nagoya University, Furo-cho, Chikusa-ku, Nagoya 464-8602, Japan \\ ${ }^{4}$ Max-Planck-Institut für Astronomie, Königstuhl 17, 69117 Heidelberg, Germany \\ 5 Zentrum für Astronomie der Universität Heidelberg, Institut für Theoretische Astrophysik, Albert-Ueberle-Str. 2, \\ 69120 Heidelberg, Germany \\ ${ }^{6}$ Institut d'Astrophysique Spatiale, CNRS, UMR8617, 91405 Orsay, France \\ 7 Sterrenkundig Observatorium, Universiteit Gent, Krijgslaan 281 S9, 9000 Gent, Belgium \\ ${ }^{8}$ UK ALMA Regional Centre Node, Jodrell Bank Centre for Astrophysics, School of Physics \& Astronomy, \\ University of Manchester, Oxford Road, Manchester M13 9PL, UK \\ 9 Laboratoire d'Astrophysique de Marseille - LAM, Université d'Aix-Marseille \& CNRS, UMR7326, 38 rue F. Joliot-Curie, \\ 13388 Marseille Cedex 13, France \\ 10 Max Planck für Extraterrestrische Physik, Giessenbachstr. 1, 85748 Garching, Germany \\ 11 Department of Physics and Astronomy, University of Sussex, Brighton, BN1 9QH, UK \\ 12 Instituto di Astrofisica e Planetologia Spaziali, INAF-IAPS, via Fosso del Cavaliere 100, 00133 Roma, Italy
}

Received 5 October 2013 / Accepted 9 December 2013

\section{ABSTRACT}

\begin{abstract}
Aims. The goal of this paper is to analyse the behaviour of the gas-to-dust mass ratio (G/D) of local Universe galaxies over a wide metallicity range. We especially focus on the low-metallicity part of the G/D vs metallicity relation and investigate several explanations for the observed relation and scatter.

Methods. We assembled a total of 126 galaxies, covering a 2 dex metallicity range and with $30 \%$ of the sample with $12+\log (\mathrm{O} / \mathrm{H}) \leq$ 8.0. We homogeneously determined the dust masses with a semi-empirical dust model including submm constraints. The atomic and molecular gas masses have been compiled from the literature. We used two $X_{\mathrm{CO}}$ scenarios to estimate the molecular gas mass: the Galactic conversion factor, $X_{\mathrm{CO}, \mathrm{MW}}$, and a $X_{\mathrm{CO}}$ that depends on the metallicity $X_{\mathrm{CO}, Z}\left(\propto Z^{-2}\right)$. We modelled the observed trend of the G/D with metallicity using two simple power laws (slope of -1 and free) and a broken power law. Correlations with morphological type, stellar masses, star formation rates, and specific star formation rates are also discussed. We then compared the observed evolution of the G/D with predictions from several chemical evolution models and explored different physical explanations for the observed scatter in the G/D values.

Results. We find that out of the five tested galactic parameters, metallicity is the main physical property of the galaxy driving the observed G/D. The G/D versus metallicity relation cannot be represented by a single power law with a slope of -1 over the whole metallicity range. The observed trend is steeper for metallicities lower than $\sim 8.0$. A large scatter is observed in the G/D values for a given metallicity: in metallicity bins of $\sim 0.1 \mathrm{dex}$, the dispersion around the mean value is $\sim 0.37$ dex. On average, the broken power law reproduces the observed G/D best compared to the two power laws (slope of -1 or free) and provides estimates of the G/D that are accurate to a factor of 1.6. The good agreement of observed values of the G/D and its scatter with respect to metallicity with the predicted values of the three tested chemical evolution models allows us to infer that the scatter in the relation is intrinsic to galactic properties, reflecting the different star formation histories, dust destruction efficiencies, dust grain size distributions, and chemical compositions across the sample.

Conclusions. Our results show that the chemical evolution of low-metallicity galaxies, traced by their G/D, strongly depends on their local internal conditions and individual histories. The large scatter in the observed G/D at a given metallicity reflects the impact of various processes occurring during the evolution of a galaxy. Despite the numerous degeneracies affecting them, disentangling these various processes is now the next step.
\end{abstract}

Key words. evolution - galaxies: dwarf - galaxies: evolution - galaxies: ISM - infrared: ISM - dust, extinction

\section{Introduction}

Metallicity is a key parameter in studying the evolution of galaxies because it traces metal enrichment. Elements are injected by

\footnotetext{
* Appendices are available in electronic form at http://www . aanda.org
}

stars in the interstellar medium (ISM) via stellar winds and/or supernovae (SN) explosions (Dwek \& Scalo 1980) and become available for the next generation of stars. The metallicity a priori traces the history of the stellar activity of a galaxy, i.e., the number of stellar generations already produced. Metallicity is thus expected to increase with age as the galaxy undergoes 
chemical enrichment through successive star formation events. However, this metal enrichment is in fact a more complex process and depends on external and internal processes occurring during galaxy evolution. Indeed, the gas phase abundance can be affected by metal-poor gas inflows that will dilute the ISM (Montuori et al. 2010; Di Matteo et al. 2011) and decrease the metallicity of the galaxy or by outflows driven by stellar feedback (stellar winds or SN shocks; e.g., Dahlem et al. 1998; Frye et al. 2002) that will eject metal-rich gas into the intergalactic medium, again resulting in a galaxy whose metallicity does not simply increase with time. Elements are also processed by gas and dust in the ISM. Dust grains form from the available metals in the ISM, and those metals can thus be depleted from the gas phase (Savage \& Sembach 1996; Whittet 2003). Metals are returned to the gas phase when dust is destroyed (e.g., by SN blast waves, Jones et al. 1994, 1996). The gas-to-dust mass ratio (G/D) links the amount of metals locked up in dust with that still present in the gas phase and is thus a powerful tracer of the evolutionary stage of a galaxy. Investigations of the relation between the observed G/D and metallicity can thus place strong constraints on the physical processes governing galaxy evolution and, more specifically, on chemical evolution models. Because of their low metallicity, dwarf galaxies can be considered as chemically young objects that are at an early stage in their evolution. In this picture, they can be seen as our closest analogues of the primordial environments present in the early Universe, from which the present-day galaxies formed. G/D of dwarf galaxies are thus crucial for constraining chemical evolution models at low metallicities.

The observed G/D of integrated galaxies as a function of metallicity has been intensively studied over the past decades (e.g., Issa et al. 1990; Lisenfeld \& Ferrara 1998; Hirashita et al. 2002; James et al. 2002; Hunt et al. 2005; Draine et al. 2007; Engelbracht et al. 2008; Galliano et al. 2008; Muñoz-Mateos et al. 2009; Bendo et al. 2010; Galametz et al. 2011; Magrini et al. 2011). In the disk of our Galaxy, the proportion of heavy elements in the gas and in the dust has been shown to scale with the metallicity (Dwek 1998) if one assumes that the time dependence of the dust formation timescale is the same as that of the dust destruction timescale. This results in a constant dust-tometal mass ratio and gives a dependence of the G/D on metallicity as $G / D \propto Z^{-1}$ (that we call hereafter the "reference" trend). This reference trend between G/D and metallicity seems consistent with the observations of galaxies with near-solar metallicities (e.g., James et al. 2002; Draine et al. 2007; Bendo et al. 2010; Magrini et al. 2011). However, some studies also show that the G/D of some low-metallicity dwarf galaxies deviate from this reference trend, with a higher G/D than expected for their metallicity (Lisenfeld \& Ferrara 1998; Galliano et al. 2003, 2005, 2008, 2011; Hunt et al. 2005; Bernard et al. 2008; Engelbracht et al. 2008; Galametz et al. 2011).

The G/D is often used to empirically estimate the "CO-free" molecular gas. This molecular gas, not directly traced by $\mathrm{CO}$ measurements, was first proposed in low-metallicity galaxies using the $158 \mu \mathrm{m}$ [CII] line (Poglitsch et al. 1995; Israel et al. 1996; Madden et al. 1997, 2012). Alternatively, using the dust mass determined from far-infrared (FIR) measurements and assuming a $\mathrm{G} / \mathrm{D}$, given the metallicity of the galaxy, a gas mass can be estimated. This gas mass is then compared to the observed HI and CO gas masses to estimate the "CO-free" gas mass. This method is also used to estimate the CO-to-H2 conversion factor in local (e.g., Guelin et al. 1993, 1995; Neininger et al. 1996; Boselli et al. 2002; Sandstrom et al. 2013) and high- $z$ galaxies (e.g., Magdis et al. 2012; Magnelli et al. 2012). However, using these methods requires an accurate estimation of the G/D at a given metallicity.

A certain number of instrumental limitations and/or model caveats have limited former studies of the G/D. First, limits in wavelength coverage in the FIR have hampered precise determination of the dust masses. For the earliest studies, the dust masses were derived from IRAS or Spitzer measurements, not extending further than $100-160 \mu \mathrm{m}$, therefore not tracing the cooler dust. As the bulk of the dust mass in galaxies often resides in the cold dust component, this has strong consequences for the dust mass and the G/D determination. Before Herschel, using Spitzer and ground-based data, Galametz et al. (2011) indeed showed that a broad wavelength coverage of the FIR-to-submillimetre (submm) part of the spectral energy distribution (SED) was critical to obtain accurate estimates of the dust masses. Second, some of the studies presented previously used modified blackbody models to derive dust masses. Using Herschel data and a semi-empirical dust model, Dale et al. (2012) showed that the dust mass modelled with a modified blackbody could be underestimated by a factor of $\sim 2$. Bianchi (2013) showed, on the contrary, that a modified blackbody modelling, using a fixed emissivity index, provides a good estimate of the dust mass. However, the case of low-metallicity dwarf galaxies has not been investigated yet and the two dust mass estimates may not be consistent with each other at low metallicities. And finally, the limited sensitivities of the pre-Herschel era instruments only allowed the detection of dust for the brightest and highest-metallicity dwarf galaxies, limiting G/D studies to metallicities $\geq 1 / 5 Z_{\odot}^{1}(12+\log (\mathrm{O} / \mathrm{H})=8.0)$.

In this work we investigate the relation between the G/D and metallicity avoiding the limitations and caveats mentioned in the previous paragraph. Using new Herschel data to constrain a semi-empirical dust model, we have a more accurate determination of the dust masses comparing to Spitzer-only dust masses and/or modified blackbody dust masses. Our sample also covers a wide range in metallicity $(2$ dex, from $12+\log (\mathrm{O} / \mathrm{H})=$ 7.1 to 9.1 ), with a significant fraction of the sample below $12+\log (\mathrm{O} / \mathrm{H})=8.0(\sim 30 \%$, see Sect. 2$)$, thanks to the increased sensitivity of Herschel which enables us to access the dust in the lowest metallicity galaxies. We are thus able to provide better constraints on the G/D at low metallicities.

In Sect. 2, we describe the sample and the method used to estimate the dust and total gas masses. Then we investigate the relation of the observed $\mathrm{G} / \mathrm{D}$ with metallicity and other galactic parameters in Sect. 3 and fit several empirical relations to the data. We then interpret our results with the aid of several chemical evolution models in Sect. 4. Throughout we consider $(G / D)_{\odot}=162^{2}$ (Zubko et al. 2004).

\section{Sample and masses}

\subsection{Sample}

We combine 3 different samples for our study of the G/D: the Dwarf Galaxy Survey (DGS, Madden et al. 2013), the KINGFISH survey (Kennicutt et al. 2011) and a subsample of the sample presented in Galametz et al. (2011, called the "G11 sample" hereafter). The basic parameters for all of the galaxies

\footnotetext{
1 Throughout we assume $(\mathrm{O} / \mathrm{H})_{\odot}=4.90 \times 10^{-4}$, i.e., $12+$ $\log (\mathrm{O} / \mathrm{H})_{\odot}=8.69$, and a total solar mass fraction of metals $Z_{\odot}=0.014$ (Asplund et al. 2009).

2 This value is from Table 6 from Zubko et al. (2004) for the BAREGR-S model, which corresponds to the dust composition used for our modelling (see Sect. 2.2).
} 


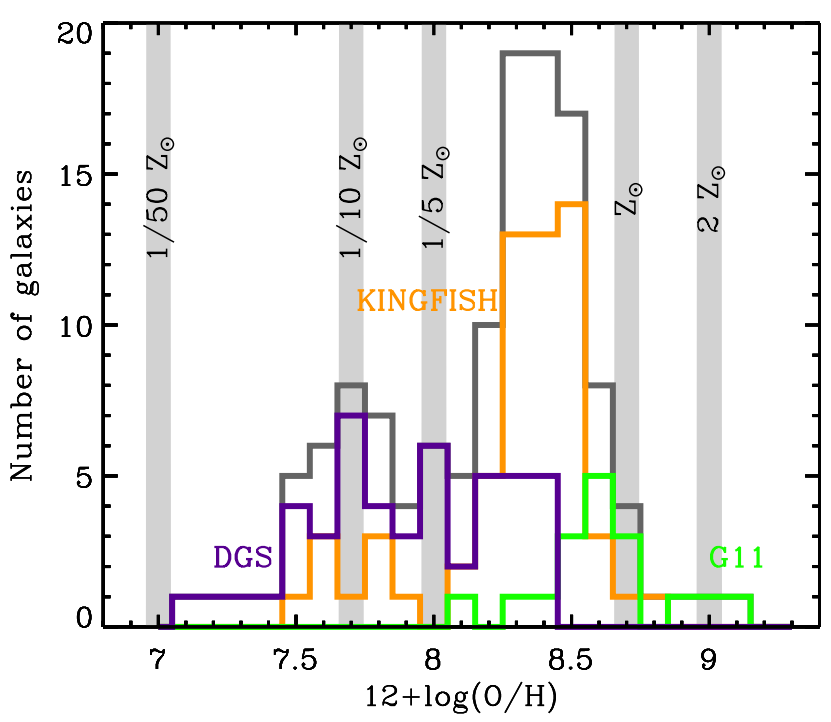

Fig. 1. Metallicity distribution of the DGS (purple), KINGFISH (orange) and $\mathrm{G} 11$ (green) samples from $12+\log (\mathrm{O} / \mathrm{H})=7.14$ to 9.10 . The total distribution is indicated in grey. Solar metallicity is indicated here as a guide to the eye, as well as the $1 / 50,1 / 10,1 / 5$ and $2 Z_{\odot}$ values.

such as positions and distances can be found in Madden et al. (2013) for the DGS; Kennicutt et al. (2011) for KINGFISH; and Galametz et al. (2011) for the G11 sample.

The DGS sample consists of 48 star-forming dwarf galaxies (mostly dwarf irregulars and blue compact dwarfs (BCDs)) covering metallicities from $12+\log (\mathrm{O} / \mathrm{H})=7.14$ to 8.43 , whereas the KINGFISH sample probes more metal-rich environments (61 galaxies including spiral, early-type and a few irregular galaxies), from $12+\log (\mathrm{O} / \mathrm{H})=7.54$ to 8.77 . The $\mathrm{G} 11$ sample consists of all of the galaxies in Galametz et al. (2011) that are neither already in the DGS nor in the KINGFISH samples, and except those galaxies which show a submm excess (see Sect. 2.2). This gives 17 additional galaxies, mostly solar or super solar environments (mostly spiral galaxies), with metallicities from $12+\log (\mathrm{O} / \mathrm{H})=8.14$ to 9.10 . The metallicity distribution for each of the 3 samples is presented in Fig. 1.

All of these metallicities have been derived using empirical strong emission line methods (see Madden et al. 2013, for the DGS, Kennicutt et al. 2011 for KINGFISH and Galametz et al. 2011 for G11 metallicity determination). The DGS and KINGFISH metallicities have been obtained through the $R_{23}$ ratio $^{3}$ with the Pilyugin \& Thuan (2005) calibration. Galametz et al. (2011) do not indicate precisely which calibration they use to convert $R_{23}$ into metallicity, thus several metallicities for the G11 galaxies were re-estimated from the original line intensities, available in the literature, with the Pilyugin \& Thuan (2005) calibration. We also assume a conservative $0.1 \mathrm{dex}$ uncertainty for the G11 metallicities. On average for the total sample, the uncertainty on the metallicity measurements is $\sim 0.1$ dex. The metallicities for the whole sample are listed in Table A.1. Other methods exist to determine metallicities and can lead to very different values, but this will only introduce a systematic offset in the adopted values here (Kewley \& Ellison 2008). Note that these metallicity values correspond to global estimates. On smaller scales within galaxies, differences can occur due to inhomogeneous mixing of metals: metallicity gradients have been observed in large spiral

${ }^{3} R_{23}=([\mathrm{OII}] \lambda 3727+[\mathrm{OIII}] \lambda \lambda 4959,5007) / \mathrm{H} \beta$. galaxies (Garnett et al. 2004; Bendo et al. 2010; Moustakas et al. 2010). Dwarf galaxies, however, are smaller in size than metalrich galaxies and we can presume, for this study, that metallicity is more homogeneous within these environments (Revaz \& Jablonka 2012; Valcke et al. 2008).

This gives us a total of 126 galaxies spanning a 2 dex range in metallicity (Fig. 1). We see that the low-metallicity end of the distribution is fairly well sampled as we have $\sim 30 \%$ of the total sample with metallicities below $1 / 5 Z_{\odot}$.

\subsection{Dust masses}

To ensure a consistent determination of the dust masses throughout our sample, all of the galaxies are modelled with the dust SED model presented in Galliano et al. (2011). It is a phenomenological model based on a two steps approach: first the modelling of one mass element of the ISM with a uniform illumination, and second the synthesis of several mass elements to account for the different illumination conditions. In the first step we assume that the interstellar radiation field (ISRF) has the spectral shape of the solar neighbourhood ISRF (Mathis et al. 1983) and use the Zubko et al. (2004) grain properties with updated PAH optical properties from Draine \& Li (2007). In the second step, we assume that the total SEDs of the galaxies can be well represented by the combination of the emission from regions with different properties. In order to do so, we assume that the dust properties are uniform and that only the illumination conditions vary in the different regions. The various regions are then combined using the Dale et al. (2001a) prescription: the distribution of the starlight intensities per unit dust mass can be represented by a power law:

$\frac{\mathrm{d} M_{\text {dust }}}{\mathrm{d} U} \propto U^{-\alpha_{U}}$ with $U_{\min } \leq U \leq U_{\min }+\Delta U$

where $M_{\text {dust }}$ is the dust mass, $U$ is the starlight intensity and $\alpha_{U}$ is the index of the power law describing the starlight intensity distribution.

We use the "standard" grain composition in our model, i.e., PAHs, silicate dust and carbon dust in form of graphite 4 . The submm emissivity index, $\beta$, for this grain composition is $\sim 2.0$ (Galliano et al. 2011). We discuss the impact of having another dust composition on our results (e.g., the use amorphous carbons instead of graphite grains) in Sect. 3.4.

The free parameters of the model are: the dust mass, $M_{\text {dust }}$, the minimum starlight intensity, $U_{\min }$, the difference between the maximum and minimum starlight intensities, $\Delta U$, the starlight intensity distribution power-law index, $\alpha_{U}$, the PAH-to-total dust mass ratio, $f_{\mathrm{PAH}}$, the mass fraction of ionised PAHs compared to the total PAH mass, $f_{\text {ion }}$, the mass fraction of very small grains (i.e., non-PAH grains with sizes $\leq 10 \mathrm{~nm}$ ), $f_{\mathrm{vsg}}$, and the contribution of the near-IR stellar continuum, $M_{\text {star }}$ (see Galliano et al. 2011, for details and a full description of the model). This model has previously been used to model dwarf galaxies, notably by Galametz et al. (2009, 2011); O'Halloran et al. (2010); Cormier et al. (2010); Hony et al. (2010); Meixner et al. (2010).

For the DGS sample, we collect IR-to-submm photometrical data from various instruments (i.e., 2MASS, Spitzer, WISE, IRAS, and Herschel) for the largest possible number of galaxies. IRS spectra are also used to constrain the MIR slope of the SEDs. For 12 DGS galaxies, the MIR continuum shape outlined by the IRS spectrum cannot be well fitted by our model. In these cases, we add an extra modified blackbody component in the

4 This corresponds to the BARE-GR-S model of Zubko et al. (2004). 
MIR, with a fixed $\beta=2.0$ and a temperature varying between 80 and $300 \mathrm{~K}$. This affect our dust masses by $\sim 5 \%$, which is well within the error bars for the dust masses $(\sim 26 \%)$, and thus this does not affect our following results. For one DGS galaxy, SBS1533+574, the impact on the dust mass is significant, but the addition of this warm modified blackbody is necessary to have a MIR-to-submm shape of the SED consistent with the observations (Rémy-Ruyer et al., in prep.) Out of the 48 DGS galaxies, only five do not have enough constraints (i.e., no observations are available or there are too many non-detections) to obtain a dust mass. Herschel data for the DGS is presented in Rémy-Ruyer et al. (2013). 2MASS, WISE and IRAS flux densities for the DGS are compiled from the NASA/IPAC IRSA databases and the literature. Spitzer-MIPS measurements are taken from Bendo et al. (2012). Spitzer-IRAC and IRS data together with a complete description of the dust modelling and the presentation of the final SEDs and dust masses for the DGS galaxies are presented in Rémy-Ruyer et al. (in prep.).

For the KINGFISH sample, we use IR-to-submm fluxes from Dale et al. $(2007,2012)$ to build the observed SEDs (i.e., observational constraints from 2MASS, Spitzer, IRAS and Herschel). The Dale et al. (2012) fluxes have been updated to the new values of the SPIRE beam areas ${ }^{5}$. The dust masses for the KINGFISH galaxies are presented in Table A.2. A submm excess is observed in some DGS and KINGFISH galaxies at $500 \mu \mathrm{m}$ (Dale et al. 2012; Rémy-Ruyer et al., in prep.). If present, the excess is rather small at $500 \mu \mathrm{m}$ and can increase as we go to longer wavelengths. However, because of the unknown origin of this excess and because of the uncertainties it can bring in the dust mass estimation, we do not attempt to model this excess with additional modifications to the model, and we thus leave aside the $500 \mu \mathrm{m}$ point in our model. Including the $500 \mu \mathrm{m}$ point results in a median difference of $\sim 3 \%$ for the dust masses in the DGS and KINGFISH samples. We discuss the influence of the presence of a submm excess in Sect. 3.4.

We also model galaxies from the G11 sample, as some model assumptions were different in Galametz et al. (2011). Herschel constraints are not present but other submm constraints are taken into account such as JCMT/SCUBA at $850 \mu \mathrm{m}$ and/or APEX/LABOCA at $870 \mu \mathrm{m}$, allowing a precise determination of the dust masses (given in Table A.2). Galametz et al. (2011) also observed a submm excess in nine galaxies of their original sample and modelled it with a very cold dust (VCD) component. However, the submm excess is not fully understood yet and this extra VCD component may lead to an overestimation of the dust mass. Because we do not have constraints between $160 \mu \mathrm{m}$ and the available ground-based submm fluxes to see where the submm excess starts, we do not consider nor model these galaxies here.

The wavelength coverage is not exactly the same from galaxy to galaxy. The most important constraints for the determination of the dust mass are constraints sampling the peak of the dust SED. Herschel provides such constraints for all of the DGS and KINGFISH galaxies. Some dwarf galaxies are not detected with Herschel at submm wavelengths (from or beyond $160 \mu \mathrm{m})$ and are noted in Fig. 4 (see Sect. 2.3). These galaxies harbour particularly warm dust (Rémy-Ruyer et al. 2013); the peak of their SED is thus shifted towards shorter wavelengths and is then well sampled by constraints until $160 \mu \mathrm{m}$ where

\footnotetext{
5 SPIRE photometer reference spectrum values:

http://herschel.esac.esa.int/twiki/bin/view/Public/ SpirePhotometerBeamProfileAnalysis: 465, 822 and 1768 square arcseconds at 250, 350, $500 \mu \mathrm{m}$ (September 2012 values).
}

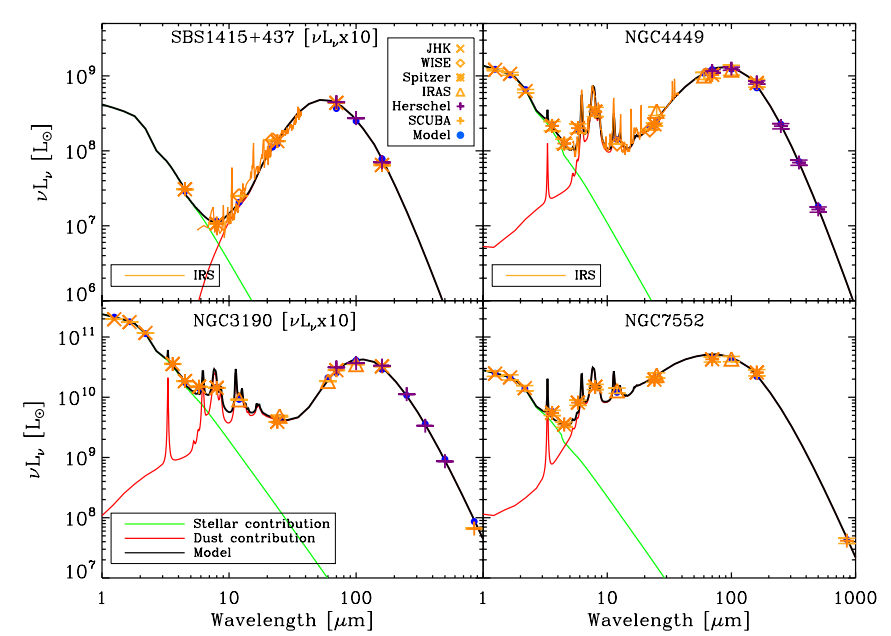

Fig. 2. Examples of SEDs modelled with the Galliano et al. (2011) model: (top left) SBS1415+437, (top right) NGC 4449, (bottom left) NGC 3190 and (bottom right) NGC 7552. The SEDs have been multiplied by 10 for SBS1415+437 and NGC 3190 for display purposes. The observed SED includes the Herschel data (purple crosses) as well as ancillary data (in orange). The different symbols code for the different instruments: Xs for 2MASS bands, diamonds for WISE, stars for Spitzer IRAC and MIPS, triangles for IRAS and orange crosses for SCUBA. The IRS spectrum is also displayed in orange for the two DGS galaxies. The total modelled SED in black is the sum of the stellar (green) and dust (red) contributions. The modelled points in the different bands are the filled blue circles.

the galaxy is still detected. For galaxies in the G11 sample, the peak of the dust SED is probed by Spitzer and/or IRAS observations and the Rayleigh-Jeans slope of the SED by longer submm wavelength observations. Thus we are confident in the dust masses we derive with these sets of constraints. As an illustration, four SEDs are presented in Fig. 2: one very lowmetallicity DGS galaxy $($ SBS $1415+437,12+\log (\mathrm{O} / \mathrm{H})=7.55)$ not observed with SPIRE, one low-metallicity DGS galaxy $(\mathrm{NGC} 4449,12+\log (\mathrm{O} / \mathrm{H})=8.20)$, and two spiral galaxies from the KINGFISH sample (NGC 3190, $12+\log (\mathrm{O} / \mathrm{H})=8.49)$ and from the G11 sample (NGC 7552, $12+\log (\mathrm{O} / \mathrm{H})=8.35)$.

The errors on the dust masses are estimated by generating 300 random realisations of the SED, perturbed according to the random and systematic noise, in order to get a distribution for the dust mass. The error bars on the dust mass are taken to be the $66.67 \%$ confidence interval of the distribution (i.e., the range of the parameter values between 0.1667 and 0.8333 of the repartition function). The detailed procedure of the error estimation is presented in Rémy-Ruyer et al. (in prep.).

\subsection{Gas masses}

Hi masses - The Hi masses and their errors are compiled from the literature, and rescaled to the distances used here. Most of the atomic gas masses are given in Galametz et al. (2011) for the G11 sample, and in Draine \& Li (2007) for the KINGFISH survey. They are presented in Madden et al. (2013) for the DGS. The errors were not available for all of the Hi measurements. When no error was available for the Hi mass, we adopted the mean value of all of the relative errors on the $\mathrm{HI}$ masses compiled from the literature: $\sim 16 \%$.

However, the Hi extent of a galaxy is not necessarily the same as the aperture used to probe the dust SED, as the HI often extends beyond the optical radius of a galaxy (Hunter 1997). 
This can be particularly true for dwarf galaxies where the Hi halo can be very extended: some irregular galaxies present unusually extended Hi gas (up to seven times the optical radius, Huchtmeier 1979; Huchtmeier et al. 1981; Carignan \& Beaulieu 1989; Carignan et al. 1990; Thuan et al. 2004). We also note that in some galaxies (e.g., NGC 4449), gas morphology may be highly perturbed due to past interactions or mergers (e.g., Hunter et al. 1999). This may also lead to significant uncertainties in the Hi mass and thus on the derived G/D (e.g., Karczewski et al. 2013). Thus we check the literature for the DGS sample for the size of the HI halo to compare it to the dust aperture. It was not possible to find this information for $\sim 38 \%$ of the sample (HI not detected or no map available). For the rest, $25 \%$ of the DGS galaxies have a Hi extent that corresponds to the dust IR aperture, which has been chosen to be 1.5 times the optical radius (for most cases, see Rémy-Ruyer et al. 2013); and 35\% have a Hi halo that is more extended. If we assume that the Hi mass distribution follows a Gaussian profile (based on the observed high central gas concentration seen in BCDs, e.g., van Zee et al. 1998, 2001; Simpson \& Gottesman 2000), we can correct the total Hi mass for these galaxies to find the Hi mass corresponding to the dust aperture. In reality, the Hi profile can show a complicated structure with clumps and shells, rendering the profile more assymetric.

Our correction corresponds to a factor of $\sim 1.55$ on average, for these galaxies. Several studies (Thuan \& Martin 1981; Swaters et al. 2002; Lee et al. 2002; Begum \& Chengalur 2005; Pustilnik \& Martin 2007) have tried to quantify the extent of the Hi halo for dwarf galaxies and found that the ratio of Hi size to the optical size is typically 2 , which gives a correction of $\sim 1.4$. The atomic gas masses for the sample, after correction if needed, are presented in Table A.1.

$\mathrm{H}_{2}$ masses - The $\mathrm{H}_{2}$ masses have been compiled from the literature. They have been rescaled, when necessary, to the distance adopted here to derive the dust masses.

The molecular gas mass is usually derived through CO measurements as $\mathrm{H}_{2}$ is not directly observable. There are two main issues in determining the molecular gas masses. First, detection of $\mathrm{CO}$ in low-metallicity galaxies is challenging: sensitivity has limited $\mathrm{CO}$ detections to galaxies with $12+\log (\mathrm{O} / \mathrm{H}) \gtrsim 8.0$ (e.g., Leroy et al. 2009; Schruba et al. 2012). The other issue in the $\mathrm{H}_{2}$ mass determination is the choice of the conversion factor between $\mathrm{CO}$ intensities and molecular gas masses, $X_{\mathrm{CO}}$. Indeed the variation of this factor with metallicity is poorly constrained, and a number of studies have been dedicated to quantifying the dependence of $X_{\mathrm{CO}}$ on metallicity (Wilson 1995; Israel 1997; Boselli et al. 2002; Israel et al. 2003; Strong et al. 2004; Leroy et al. 2011; Schruba et al. 2012; Bolatto et al. 2013). From a sample of 16 dwarf galaxies, and assuming a constant $\mathrm{H}_{2}$ depletion timescale, Schruba et al. (2012) found a $X_{\mathrm{CO}}$ scaling with $(\mathrm{O} / \mathrm{H})^{-2}$. This relation takes into account possible "CO-free" gas as the $X_{\mathrm{CO}}$ conversion factor is estimated from the total reservoir of molecular gas needed for star formation (Schruba et al. 2012). Following Cormier et al. (2014), we estimate the molecular gas masses from a constant $X_{\mathrm{CO}}$ factor using the Galactic value: $X_{\mathrm{CO}, \mathrm{MW}}=2.0 \times 10^{20} \mathrm{~cm}^{-2}\left(\mathrm{~K} \mathrm{~km} \mathrm{~s}^{-1}\right)^{-1}$ (Ackermann et al. 2011), giving us $M_{\mathrm{H}_{2}, \mathrm{MW}}$, and from a $X_{\mathrm{CO}}$ factor depending on $(\mathrm{O} / \mathrm{H})^{-2}: X_{\mathrm{CO}, Z}$, giving us $M_{\mathrm{H}_{2}, Z}$. This provides a conservative range of molecular gas mass estimates that reflects how uncertain the molecular gas mass determination is. For this reason, we do not give any error bars on our molecular gas masses.

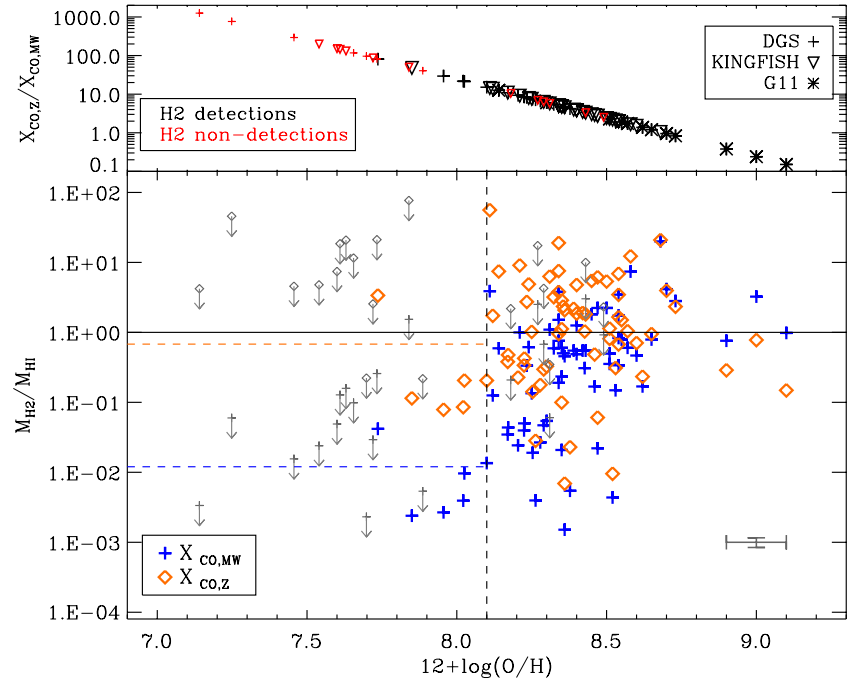

Fig. 3. Bottom: $M_{\mathrm{H}_{2}} / M_{\mathrm{HI}}$ as a function of metallicity for the whole sample. The blue crosses are for molecular gas masses computed with $X_{\mathrm{CO}, \mathrm{MW}}$ and the orange diamonds are for molecular gas masses computed with $X_{\mathrm{CO}, Z}$. Upper limits in the molecular gas mass are indicated with grey arrows and smaller grey symbols. The mean error for the data points is shown in grey on the bottom right of the plot. The plain line shows the unity line. The dashed blue and orange lines show the $1.2 \%$ and $68 \%$ molecular-to-atomic gas mass fractions respectively and represent the mean $\mathrm{H}_{2}$-to-Hi ratio of the detected galaxies with $12+\log (\mathrm{O} / \mathrm{H})<8.1$ (see text). The horizontal dashed black line shows the metallicity threshold $12+\log (\mathrm{O} / \mathrm{H})=8.1$ to guide the eye. Top: $X_{\mathrm{CO}, \mathrm{Z}} / X_{\mathrm{CO}, \mathrm{MW}}$ illustrating the $(\mathrm{O} / \mathrm{H})^{-2}$ dependence adopted to compute $X_{\mathrm{CO}, Z}$. The symbols delineate between the three samples: crosses, downward triangles and stars for the DGS, KINGFISH and G11 samples respectively. The colours differentiate between $\mathrm{H}_{2}$ detections (in black, corresponding to the coloured points on the bottom panel) and the $\mathrm{H}_{2}$ non-detections (in red, corresponding to the grey points in the bottom panel).

In order to go beyond the $\mathrm{CO}$ upper limits and to constrain the $\mathrm{G} / \mathrm{D}$ behaviour at low metallicities we find a way to estimate the amount of molecular gas for the lowest metallicity galaxies. Figure 3 shows the ratio of $M_{\mathrm{H}_{2}}$-to- $M_{\mathrm{HI}}$ as a function of metallicity for our sample and for both cases of $X_{\mathrm{CO}}$. We note that around $12+\log (\mathrm{O} / \mathrm{H}) \sim 8.1$ the ratio $M_{\mathrm{H}_{2}} / M_{\mathrm{HI}}$ drops suddenly for the detected galaxies for both $X_{\mathrm{CO}}$. For these very low-metallicity galaxies with $12+\log (\mathrm{O} / \mathrm{H}) \leq 8.1$, the mean ratio between the detected $M_{\mathrm{H}_{2}}$ and $M_{\mathrm{HI}}$ is $1.2 \%$, for $X_{\mathrm{CO}, \mathrm{MW}}$. Using $X_{\mathrm{CO}, Z}$, this ratio goes up to $68 \%$. Thus for galaxies with non-detections in $\mathrm{CO}$ or without any $\mathrm{CO}$ observations, and with $12+\log (\mathrm{O} / \mathrm{H}) \leq 8.1$, we replace the upper limit values by $0.012 \times M_{\mathrm{HI}}$ for $M_{\mathrm{H}_{2}, \mathrm{MW}}$ and $0.68 \times M_{\mathrm{HI}}$ for $M_{\mathrm{H}_{2}, Z}$. Given the low molecular gas fraction we find, this will not greatly affect our interpretation of G/D nor the conclusions. From now on, the galaxies for which we apply this correction will be treated as detections. This $12+\log (\mathrm{O} / \mathrm{H})$ value of $\sim 8.1$ has already been noted as being special for dwarf galaxies (e.g., for the strength of the PAH features: Engelbracht et al. 2005, 2008; Madden et al. 2006; Draine et al. 2007; Galliano et al. 2008). The molecular gas masses we use in the following analysis are presented in Table A.1.

Total gas masses - We get the total gas mass, $M_{\text {gas }}$, by adding all of the different gas contributions: the atomic gas mass, the molecular gas mass, the helium gas mass and the gaseous metal mass:

$M_{\mathrm{gas}}=M_{\mathrm{HI}}+M_{\mathrm{H}_{2}}+M_{\mathrm{He}}+Z_{\mathrm{gal}} \times M_{\mathrm{gas}}$, 
a)

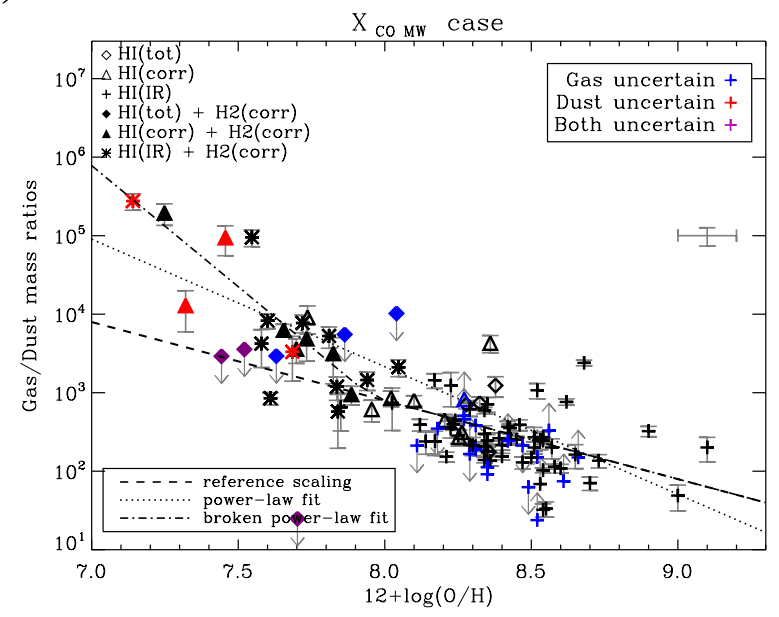

c)

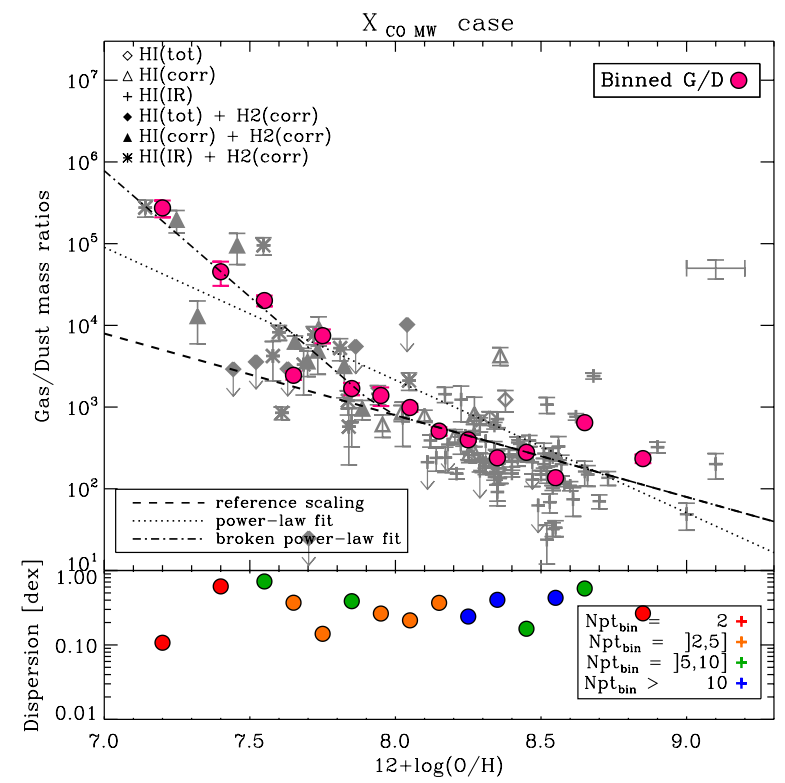

b)

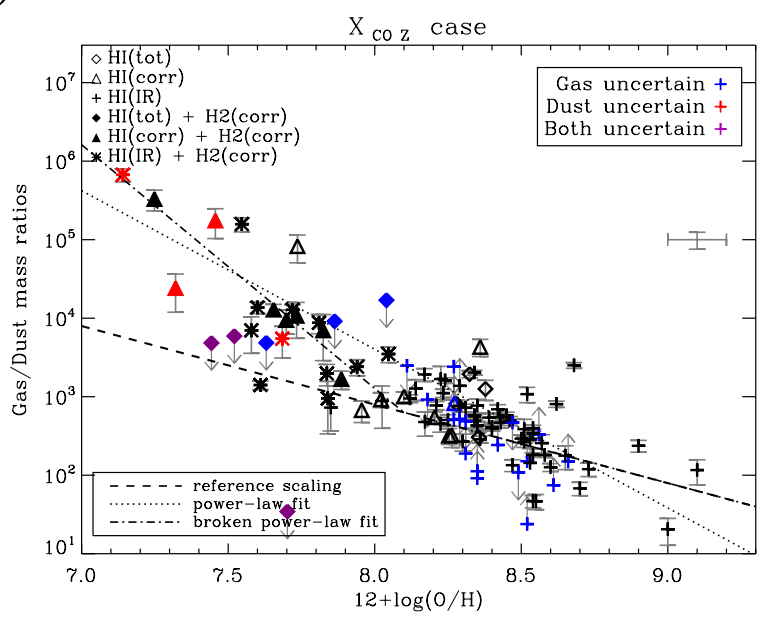

d)

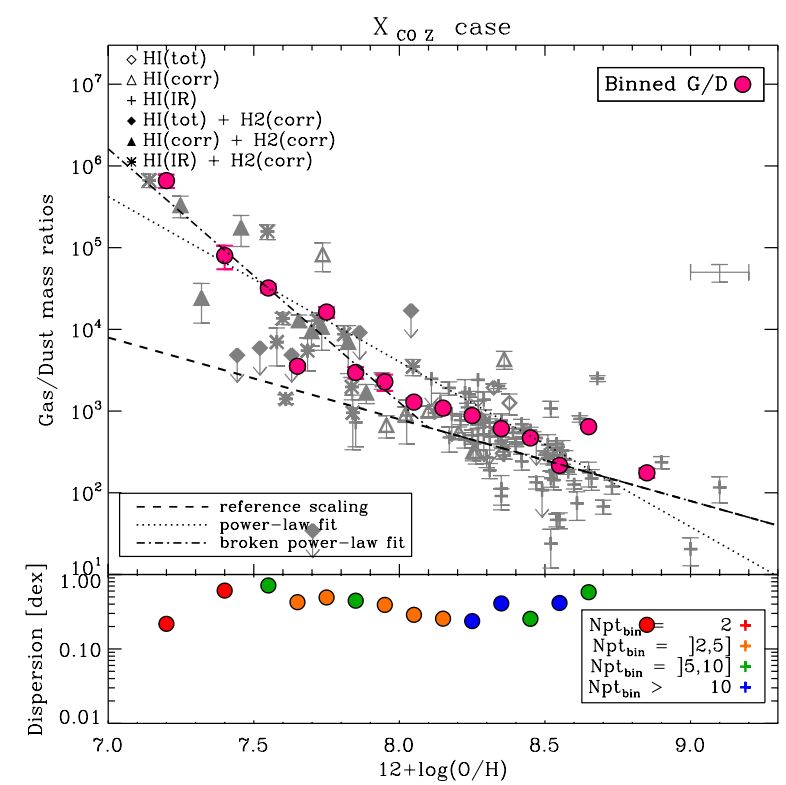

Fig. 4. Top row: G/D as a function of metallicity for the 2 values of $X_{\mathrm{CO}}: X_{\mathrm{CO}, \mathrm{Mw}} \mathbf{a}$ ) and $\left.X_{\mathrm{CO}, Z} \mathbf{b}\right)$. The mean error for the data points is shown in grey on the right of the plots. The colours code the reliability of the point depending whether the gas mass is uncertain (in blue), the dust mass is uncertain (in red) or if both are uncertain (in purple). The symbol traces the changes made in the $\mathrm{HI}_{\mathrm{I}}$ and $\mathrm{H}_{2}$ masses (see text for details on the uncertainties and the changes on the gas masses). The dashed line represents the reference scaling of the G/D with metallicity (not fit to the data). The dotted and dash-dotted lines represent the best power-law and best broken power-law fits to the data. Bottom row: same as top row for $X_{\mathrm{CO}, \mathrm{Mw}}$ c) and $X_{\mathrm{CO}, Z} \mathbf{d}$ ), where the binned G/D values (see text) have been added as pink filled circles. For clarity, the observed G/D values are now shown in grey. On the bottom panels the relative dispersion in each bins, in terms of standard deviation, is shown and the colours show the number of galaxies in each bin.

where $M_{\mathrm{He}}$ is the helium mass and $Z_{\mathrm{gal}}$ the mass fraction of metals in the galaxy. Assuming $M_{\mathrm{He}}=Y_{\odot} M_{\text {gas }}$, where $Y_{\odot}$ is the Galactic mass fraction of Helium, $Y_{\odot}=0.270$ (Asplund et al. 2009), we have:

$M_{\mathrm{gas}}=\mu_{\mathrm{gal}}\left(M_{\mathrm{HI}}+M_{\mathrm{H}_{2}}\right)$,

with $\mu_{\text {gal }}=1 /\left(1-Y_{\odot}-Z_{\text {gal }}\right)$ the mean atomic weight. $\mu_{\text {gal }}$ has been computed for each galaxy and the mean value for our sample is $1.38 \pm 0.01$ (see Table A.1). We get $Z_{\text {gal }}$ assuming $\left(Z_{\text {gal }} / Z_{\odot}\right)=$ $\left(\mathrm{O} / \mathrm{H}_{\mathrm{gal}}\right) /\left(\mathrm{O} / \mathrm{H}_{\odot}\right)$ and $Z_{\odot}=0.014$ (Asplund et al. 2009).

We assume here that the ionised gas mass $\left(M_{\mathrm{HII}}\right)$ is negligible compared to the Hi mass. We perform the test for 67 galaxies of the sample, with $M_{\mathrm{HII}}$ derived from $\mathrm{H} \alpha$ measurements of Gil de Paz et al. (2003); Kennicutt et al. (2009); Skibba et al. (2011) and found $M_{\mathrm{HII}} / M_{\mathrm{HI}} \sim 0.2 \%$. However, we found two dwarf galaxies for which the ionised gas mass should be taken into account as it contributes equally or more than the atomic gas mass: Haro11 $\left(M_{\mathrm{HII}} \sim 1.2 \times M_{\mathrm{HI}}\right.$, Cormier et al. 2012) and Pox $186\left(M_{\mathrm{HII}} \sim M_{\mathrm{HI}}\right.$, Gil de Paz et al. 2003). For these two galaxies, the total gas mass also includes $M_{\mathrm{HII}}$.

The G/D as a function of metallicity is presented in Figs. $4 \mathrm{a}, \mathrm{b}$ for the two cases: $X_{\mathrm{CO}, \mathrm{Mw}}$ or $X_{\mathrm{CO}, \mathrm{Z}}$. The average error on the observed G/D is $\sim 27 \%$ in both $X_{\text {CO }}$ cases $(\sim 10 \%$ for the total gas mass and $\sim 26 \%$ for the dust mass). The dashed line indicates the reference scaling of the G/D with metallicity. The colours of the symbols indicate the reliability of the data points by tracing if the gas or dust masses determinations are uncertain. Blue symbols refer to $\mathrm{HI}$ or $\mathrm{H}_{2}$ non-detections or to the absence of $\mathrm{H}_{2}$ observations for the galaxy. Red symbols indicate that the galaxy is not detected at wavelengths $\geq 160 \mu \mathrm{m}$. 
The combination of both indications for the gas and dust masses is shown with the purple symbols. Black symbols indicate that both gas and dust masses have reliable measurements $(67 \%$ of the sample).

The type of symbols indicates whether or not the Hi and/or $\mathrm{H}_{2}$ masses have been corrected. For the $\mathrm{HI}_{\mathrm{I}}$ masses we distinguish three cases for the DGS galaxies: the Hi extent of the galaxy is unknown and we cannot correct the Hi mass (diamonds), the Hi extent is known and greater than the dust aperture and we correct the Hi mass (triangles) and the Hi extent is known and similar to the dust aperture, there is no need to correct the Hi mass (crosses). The galaxies with $12+\log (\mathrm{O} / \mathrm{H})<8.1$ for which the $\mathrm{H}_{2}$ masses have been corrected (either from upper limit or lack of measurements) are indicated as filled symbols (see paragraph above on $\mathrm{H}_{2}$ masses).

\section{Analysis}

\subsection{Observed gas-to-dust mass ratio - metallicity relation and dispersion}

To evaluate the general behaviour and scatter in the G/D values at different metallicities, we consider the error-weighted mean values of $\log (\mathrm{G} / \mathrm{D})$ in metallicity bins (neglecting the upper/lower limits), with the bin sizes chosen to include at least two galaxies and to span at least 0.1 dex. The result is overlaid as pink filled circles in Figs. 4c, d. We also look at the dispersion of the G/D values in each metallicity bin (see bottom panels of Figs. 4c, d), by computing the standard deviation of the $\log (\mathrm{G} / \mathrm{D})$ values in each bin (also neglecting the upper/lower limits). The dispersion is $\sim 0.37$ dex (i.e., a factor of 2.3) on average for all bins and for both $X_{\mathrm{CO}}$ values. Additionally, in one bin the G/D vary on average by one order of magnitude. This confirms that the relation between G/D and metallicity is not trivial even at a given metallicity, and over the whole metallicity range. We also see that the dispersion in the observed G/D values does not depend on metallicity. This indicates that the scatter within each bin may be intrinsic and does not reflect systematic observational or correction errors. This also means that the metallicity is not the only driver for the observed scatter in the G/D values: other processes operating in galaxies can lead to large variation in the G/D in a given metallicity range, throughout this range. However, there might be a selection bias in our sample. Indeed our sample is mainly composed of star-forming gas-rich dwarf galaxies at low metallicities and spiral galaxies at high metallicities (see Fig. B.2). We could wonder if gas-poor dwarf galaxies would show different, possibly lower, G/D than that observed in gas-rich dwarfs, thus possibly increasing the observed scatter at low metallicities. On the high-metallicity side, Smith et al. (2012) showed that the 30 elliptical galaxies detected with Herschel in the Herschel Reference Survey (HRS, Boselli et al. 2010) had a mean G/D of $\sim 120$, which is slightly lower than what we find for our elliptical and spiral galaxies at moderate metallicities (see Fig. B.3, the mean $\mathrm{G} / \mathrm{D}$ are 150 and 270 for spirals, and 300 and 500 for ellipticals on average for $X_{\mathrm{CO}, \mathrm{MW}}$ and $X_{\mathrm{CO}, Z}$ respectively). However, the dust masses were estimated via a modified blackbody model, thus we will not go deeper into any further comparison. Nonetheless, including more elliptical galaxies might also slightly increase the scatter at high metallicities.

\subsection{Gas-to-dust mass ratio with other galactic parameters}

In this paragraph we want to see how the G/D depends on other galactic properties, namely the morphological type, the stellar mass and the star formation rate (SFR). The distribution for each of these parameters for the sample is presented in Appendix B. We look at the variation of the G/D as a function of these three parameters and the results are shown in Fig. B.3, where the galaxies are colour coded by their metallicity.

For the morphological types, the "normal" type galaxies (i.e., elliptical and spirals) have lower G/D than irregular (dwarfs) galaxies or BCDs (Fig. B.3). As for the other two parameters, we found each time a correlation: galaxies with higher stellar masses or higher star formation rates have lower G/D than less massive or less active galaxies (Fig. B.3). However, the correlation is weaker than with the metallicity: we have Spearman rank coefficients ${ }^{6} \rho \sim-0.30$ and -0.25 between G/D and the stellar masses and the star formation rates respectively, versus $\rho \sim-0.45$ with the metallicity. On the absolute scale dwarf galaxies have lower stellar masses and lower absolute star formation rate than their metal-rich counterparts. The specific star formation rate (SSFR), defined as the SFR divided by the stellar mass, is more representative of the intrinsic star formation activity of the galaxy. When looking at the G/D as a function of SSFR, we find a even weaker correlation $(\rho \sim 0.16)$ between these two quantities (see Fig. B.1).

As all of these parameters are themselves related to the metallicity of the galaxy, the observed weaker correlations are thus "secondary" correlations, resulting from the correlation between the metallicity and the other galactic parameters. This means that, as far as these five parameters are concerned (metallicity, stellar mass, SFR, SSFR and morphological type), metallicity is the fundamental parameter driving the observed G/D values. Thus in the following, we focus only on the relation between $\mathrm{G} / \mathrm{D}$ and metallicity.

\subsection{Empirical relations and scatter}

To investigate the variation of the G/D with metallicity, we first fit a power law (dotted line in Fig. 4) through the observed G/D values (excluding the limits): $G / D \propto(\mathrm{O} / \mathrm{H})^{\alpha}$. The fit is performed with the IDL procedure MPFIT ${ }^{7}$ and is shown in Fig. 4. The fit is weighted by the individual errors bars of the G/D values and the number density of points to avoid being dominated by the more numerous high metallicity galaxies. We get a slope for the power law of $\alpha=-1.6 \pm 0.3$ for $X_{\mathrm{CO}, \mathrm{MW}}$ and $\alpha=-2.0 \pm 0.3$ for $X_{\mathrm{CO}, Z}$. In both cases, $\alpha$ is lower than -1 , which corresponds to the slope of the reference relation.

We also fit a broken power law (dash-dotted line in Fig. 4), with two slopes $\alpha_{\mathrm{L}}$ and $\alpha_{\mathrm{H}}$ to describe the low- and highmetallicity slopes respectively, and with a transition metallicity, $x_{\mathrm{t}}$, between the two regimes. Several studies (e.g., James et al. 2002; Draine et al. 2007; Galliano et al. 2008; Leroy et al. 2011) have shown that the G/D was well represented by a power law with a slope of -1 at high metallicities and down to $12+\log (\mathrm{O} / \mathrm{H}) \sim 8.0-8.2$, and thus we fix $\alpha_{\mathrm{H}}=-1$. This gives us a low-metallicity slope, $\alpha_{\mathrm{L}}$, of $-3.1 \pm 1.8$ with a transition metallicity of $7.96 \pm 0.47$ for $X_{\mathrm{CO}, \mathrm{MW}}$ and $\alpha_{\mathrm{L}}=-3.1 \pm 1.3$ and a transition around a metallicity of $8.10 \pm 0.43$ for $X_{\mathrm{CO}, Z}$. This corresponds to a predicted G/D uncertain to a factor of $\sim 1.6$. The low-metallicity slopes, $\alpha_{\mathrm{L}}$, are also for both cases lower than -1 . The parameters for the different empirical relations are given in Table 1.

\footnotetext{
6 The Spearman rank coefficient, $\rho$, indicates how well the relationship between $X$ and $Y$ can be described by a monotonic function: monotonically increasing: $\rho>0$, or monotonically decreasing: $\rho<0$.

7 http://www.physics.wisc.edu/ craigm/idl/idl.html
} 

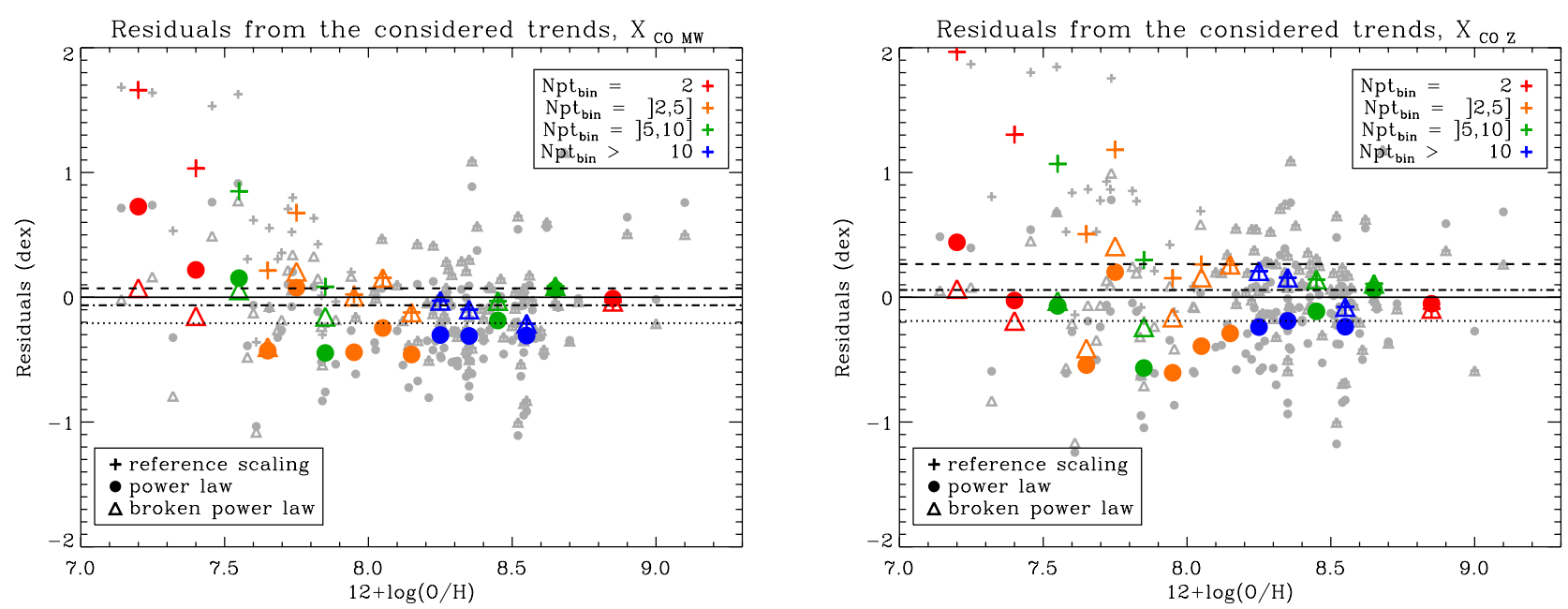

Fig. 5. Residuals (i.e., logarithmic distance) between the observed (and detected) G/D and predicted G/D from the three relations for $X_{\mathrm{CO}, \mathrm{MW}}($ left) and $X_{\mathrm{CO}, Z}($ right): reference scaling of the $\mathrm{G} / \mathrm{D}$ with metallicity (crosses), the best power-law fit (filled circles) and the best broken power-law fit (triangles). These residuals are shown in grey for the individual galaxies and in colour for the average residuals in each metallicity bin defined in Sect. 3.1. The colours show the number of galaxies in each bin. The mean residual for all of the observed G/D values is shown by the dashed (reference scaling), dotted (power-law fit) and dash-dotted (broken power-law fit) lines for the three relations and are reported in Table 1.

Table 1. Parameters for the three empirical relations between the G/D and metallicity: power law (slope of -1 and free) and broken power law for the two $X_{\mathrm{CO}}$ values.

\begin{tabular}{|c|c|c|}
\hline Parameters & $X_{\mathrm{CO}, \mathrm{MW}}$ case & $X_{\mathrm{CO}, Z}$ case \\
\hline \multicolumn{3}{|c|}{ Power law, slope fixed: $y=a+\left(x_{\odot}-x\right)$ ("reference" scaling) } \\
\hline$a^{1,2}$ & 2.21 & 2.21 \\
\hline average logarithmic distance ${ }^{3}[\mathrm{dex}]$ & 0.07 & 0.27 \\
\hline \multicolumn{3}{|c|}{ Power-law, slope free: $y=a+\alpha\left(x_{\odot}-x\right)$} \\
\hline 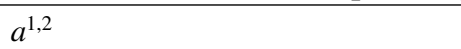 & 2.21 & 2.21 \\
\hline$\alpha$ & $1.62 \pm 0.34$ & $2.02 \pm 0.28$ \\
\hline average logarithmic distance ${ }^{3}[\mathrm{dex}]$ & -0.21 & -0.19 \\
\hline \multicolumn{3}{|c|}{$\begin{array}{l}\text { Broken power law: } \\
\begin{array}{r}y=a+\alpha_{\mathrm{H}}\left(x_{\odot}-x\right) \text { for } x>x_{\mathrm{t}} \\
y=b+\alpha_{\mathrm{L}}\left(x_{\odot}-x\right) \text { for } x \leq x_{\mathrm{t}}\end{array}\end{array}$} \\
\hline$a^{1,2}$ & 2.21 & 2.21 \\
\hline$\alpha_{\mathrm{H}}^{1}$ & 1.00 & 1.00 \\
\hline$b^{\mathrm{H}}$ & 0.68 & 0.96 \\
\hline$\alpha_{\mathrm{L}}$ & $3.08 \pm 1.76$ & $3.10 \pm 1.33$ \\
\hline$x_{\mathrm{t}}$ & $7.96 \pm 0.47$ & $8.10 \pm 0.43$ \\
\hline average logarithmic distance ${ }^{3}[\mathrm{dex}]$ & -0.06 & 0.06 \\
\hline
\end{tabular}

Notes. $y=\log (\mathrm{G} / \mathrm{D}), x=12+\log (\mathrm{O} / \mathrm{H})$ and $x_{\odot}=8.69$. $^{(1)}$ Fixed parameter. ${ }^{(2)}$ This corresponds to the solar G/D: $G / D_{\odot}=10^{a}=162$ (Zubko et al. 2004). ${ }^{(3)}$ Derived for all of the individual galaxies, neglecting the upper/lower limits on the G/D.

If we let $\alpha_{\mathrm{H}}$ free in the broken power-law fit, we get similar results within errors for $\alpha_{\mathrm{L}}$ and $x_{\mathrm{t}}$ in both $X_{\mathrm{CO}}$ cases. We get $\alpha_{\mathrm{H}}=-0.5 \pm 0.9$ and $\alpha_{\mathrm{H}}=-1.6 \pm 0.6$, for $X_{\mathrm{CO}, \mathrm{MW}}$ and $X_{\mathrm{CO}, Z}$ respectively, which is coherent with a slope of -1 within errors. Note that we have imposed here that our fits go through the solar G/D determined by Zubko et al. (2004). If we relax this condition (i.e., do not fix our " $a$ " parameter in Table 1), we get values of the solar $\mathrm{G} / \mathrm{D}$ ranging from $(G / D)_{\odot}=90$ to 240 within $\sim 60 \%$ of the value from Zubko et al. (2004).

Now we consider the deviation from each relation by looking at the logarithmic distance between the observed G/D values and the $\mathrm{G} / \mathrm{D}$ values predicted by each of the three relations presented in Table 1. This is a way to look at the residuals from the two fits and the reference scaling, even though we did not actually fit the reference trend to the G/D values. These residuals are shown in Fig. 5. Average residuals in each metallicity bin defined previously are also computed. For a given point, the best relation is the one giving the residual closest to zero. From Fig. 5 we have another confirmation that a reference scaling of the G/D with metallicity does not provide reliable estimates of the G/D at low metallicities $12+\log (\mathrm{O} / \mathrm{H}) \lesssim 8.0$. We also note that, for the average residuals, the broken power law gives the residuals that are the closest to zero for nearly all of the metallicity bins in both $X_{\mathrm{CO}, \mathrm{MW}}$ and $X_{\mathrm{CO}, Z}$ cases.

Even though $30 \%$ of our sample have metallicities below $1 / 5 Z_{\odot}$, only seven galaxies have $12+\log (\mathrm{O} / \mathrm{H}) \leq 7.5$ with two of them not detected in Hi (SBS1159+545 and Tol1214-277). The remaining five galaxies (IZw18, HS0822+3542, SBS0335052 , SBS1415+437 and UGC4483) are strong constraints for the broken power-law fit. These five galaxies all present broad dust SEDs peaking at very short wavelengths $(\sim 40 \mu \mathrm{m}$, and $\sim 70 \mu \mathrm{m}$ for HS0822+3542), indicating overall warmer dust with a wide range of dust grain temperatures, and subsequently very low dust masses; hence their high G/D. This peculiar SED shape had already been noted by Rémy-Ruyer et al. (2013).

Using Herschel data and a semi-empirical SED model, Sandstrom et al. (2013) looked at the G/D in a sub-sample of 26 KINGFISH galaxies, mostly spirals. They simultaneously derive $X_{\mathrm{CO}}$ and $\mathrm{G} / \mathrm{D}$ for their sample, taking advantage of the high spatial resolution of the KINGFISH gas and dust data. They found that the G/D for these galaxies follows the reference trend with the metallicity and shows small scatter. Their metallicity range is from $12+\log (\mathrm{O} / \mathrm{H}) \sim 8.1$ to 8.8 and thus these results are in agreement with our findings. Moreover the small scatter (less than a factor of 2) observed by Sandstrom et al. (2013) can be due to the fact that they are probing very similar environments. In our case we have a wide variety of morphological types represented in our sample, that results in a larger scatter (a factor of $\sim 5$ and 3 for $X_{\mathrm{CO}, \mathrm{MW}}$ and $X_{\mathrm{CO}, Z}$ respectively for this metallicity range).

\subsection{Discussion}

In the previous section, we have shown that the reference scaling relation between metallicity and G/D derived for metallicities 
above $12+\log (\mathrm{O} / \mathrm{H}) \sim 8.0$ does not apply to objects with lower metallicity. We empirically derived a new scaling relation better described by a broken power law with a transition metallicity around $12+\log (\mathrm{O} / \mathrm{H}) \sim 8.0$, which confirms the importance of this value in low-metallicity dwarf galaxies. As mentioned in the Introduction, this reference scaling relation arises from the hypothesis that the dust formation timescale and the dust destruction timescale behave similarly with time. Thus a possible interpretation of our results would be that the balance between formation and destruction of dust grains is altered at low metallicity, resulting in the observed steeper trend. Dwarf galaxies are subject to an overall harder ISRF than more metal-rich environments (Madden et al. 2006). The harder UV photons travel deeper into the ISM and photoprocess dust in much deeper regions in the clouds limiting the accretion and coagulation of the grains. The hard ISRF also affects the dust survivability in such extreme environments, especially carbonaceous dust: the dust destruction by hard UV photons is enhanced in low-metallicity galaxies for small carbon grains (e.g., Pilleri et al. 2012; Bocchio et al. 2012, 2013). In dwarf galaxies, dust destruction by SN shocks is enhanced too compared to larger scale galaxies, as most of the ISM can be affected by the shock due to the small physical size of the dwarfs and the globally lower density of the ISM.

In the following paragraphs we discuss the impact of several assumptions made to estimate the G/D on our results: the dust composition, the choice of the radiation field for the dust modelling, and the potential presence of a submm excess in some of our dwarf galaxies.

Dust composition - Galliano et al. (2011) demonstrated that a more emissive dust grain composition compared to that of the Galaxy, using amorphous carbon instead of graphite for the carbonaceous grains, is more consistent for the low-metallicity Large Magellanic Cloud (LMC). This result has been confirmed by Galametz et al. (2013) in a star-forming complex of the LMC with an updated version of the SPIRE calibration ${ }^{8}$. Changing accordingly the dust composition in our low-metallicity galaxies would give lower dust masses (by a factor of $\sim$ two to three in the case of the LMC) with more emissive dust grains and would increase the G/D by the same factor, increasing the discrepancy at low metallicities between the observed G/D and the predicted $\mathrm{G} / \mathrm{D}$ from the reference scaling relation.

Radiation field - In the dust modelling, we use an ISRF with the spectral shape of the Galactic ISRF for all of our galaxies for consistency. However, the ISRF in low-metallicity dwarf galaxies is harder, so we could wonder if this spectral shape is appropriate for the modelling of dwarf galaxies. The shape of the radiation field determines the emission of out-of-equilibrium small grains. Increasing the hardness of the radiation field increases the maximum temperature the small grains can reach when they undergo stochastic heating. However, these very small grains only have a minor contribution to the total dust mass, and thus the assumed shape of the ISRF does not bias our estimation of the total dust mass for dwarf galaxies.

Submm excess - A submm excess has been observed in the past in several low-metallicity galaxies that current dust SED

\footnotetext{
8 This updated SPIRE calibration from September 2012 had the effect of decreasing the SPIRE flux densities by about $10 \%$ compared to the Galliano et al. (2011) study.
}

models are unable to fully explain (Galliano et al. 2003, 2005; Dumke et al. 2004; Bendo et al. 2006; Zhu et al. 2009; Galametz et al. 2009, 2011; Bot et al. 2010; Grossi et al. 2010). Several hypotheses have been made to explain this excess among which the addition of a very cold dust (VCD) component in which most of the dust mass should reside. This VCD component would be in the form of very dense clumps in the ISM (Galliano et al. 2003, 2005). Taking this additional VCD component into account in the DGS and KINGFISH galaxies presenting a submm excess can result in a drastic increase of the dust mass and thus in a lower G/D. However, Galliano et al. (2011) showed for a strip of the LMC that the submm excess is more significant in the diffuse regions, possibly in contradiction with the hypothesis of very cold dust in dense clumps. Other studies have suggested an enhanced fraction of very small grains with high emissivity (Lisenfeld et al. 2002; Dumke et al. 2004; Bendo et al. 2006; Zhu et al. 2009), "spinning" dust emission (Ysard \& Verstraete 2010) or emission from magnetic nano-particles (Draine \& Hensley 2012) to explain the submm excess. Meny et al. (2007) proposed variations of the optical properties of the dust with the temperature which results in an enhanced emission of the dust at submm/mm wavelengths. Galametz et al. (2013) demonstrated that an amorphous carbon dust composition did not lead to any submm excess in the LMC. This alternative dust composition is thus also a plausible explanation for the submm excess. As for the discussion on the dust composition, using dust masses estimated with amorphous carbon grains for galaxies presenting a submm excess would result in an increase of a factor of $\sim 2$ for the $\mathrm{G} / \mathrm{D}$ of these galaxies.

\section{Chemical evolution models}

Chemical evolution models, under certain assumptions, can predict a possible evolution of the G/D as metallicity varies in a galaxy. For example, in the disk of our Galaxy, chemical evolution models predict this "reference" scaling of the G/D with the metallicity (Dwek 1998). We consider three different models here, from Galliano et al. (2008), Asano et al. (2013a) and Zhukovska (2014) to interpret our data. However, we have to keep in mind during this comparison that, since we do not know the ages of these galaxies and that the same metallicity can be reached at very different times by different galaxies, our sample cannot be considered as the evolution (snapshots) of one single galaxy.

\subsection{A simple model to begin with}

Galliano et al. (2008) developed a one-zone single-phase chemical evolution model, based on the model by Dwek (1998). They consider a closed-box model where the evolution of the dust content is regulated by balancing dust production by stars and dust destruction by star formation and SN blast waves. They assume full condensation of the elements injected by Type II supernovae (SNII) into dust and instantaneous mixing of the elements in the ISM. The model is shown in Fig. 6 for various SN destruction efficiencies as the dark grey zone.

Two things can be noticed from Fig. 6. First, the model is fairly consistent with the observed G/D at high metallicities within the scatter, and down to metallicities $\sim 0.5 Z_{\odot}$. Second, the model does not work at low metallicities and systematically underestimates the G/D. This has already been noted by Galliano et al. (2008) for their test sample of galaxies and was attributed to the very crude assumptions made in the modelling, especially the instantaneous mixing of the SNII elements in the ISM. Another 

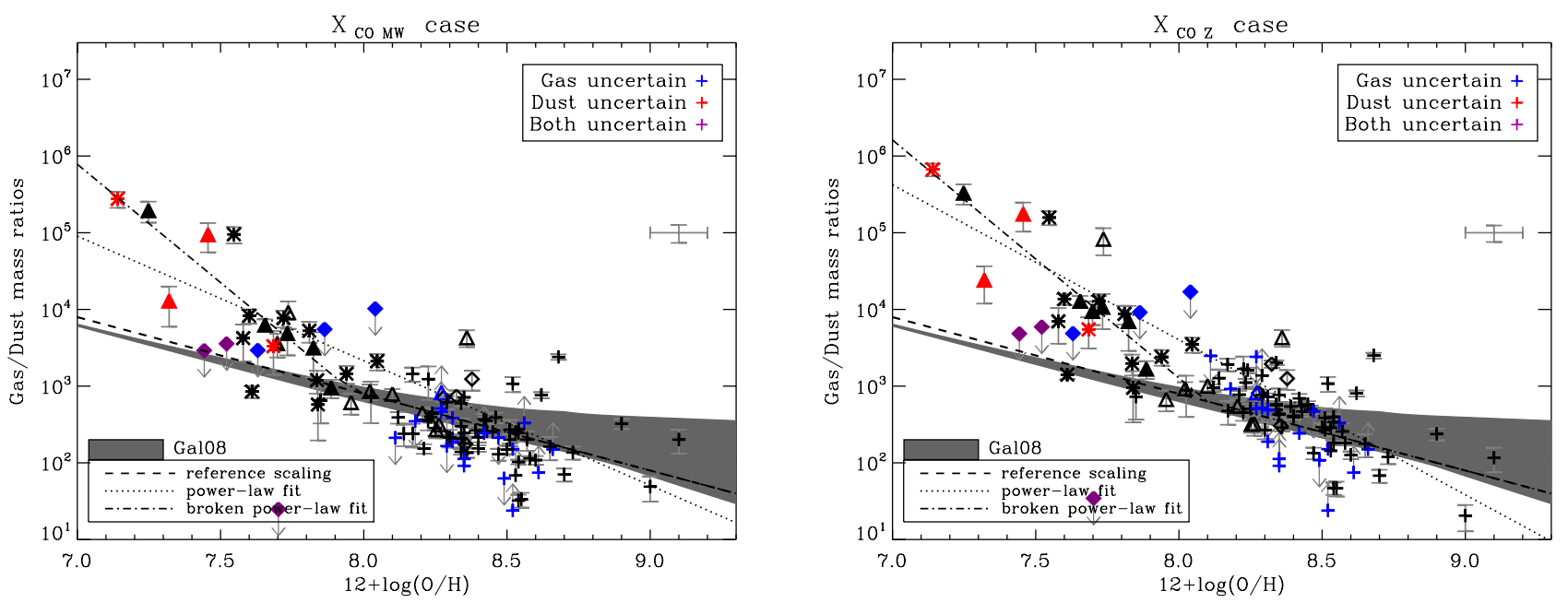

Fig. 6. G/D as a function of metallicity for the 2 values of $X_{\mathrm{CO}}: X_{\mathrm{CO}, \mathrm{MW}}(l e f t)$ and $X_{\mathrm{CO}, Z}(r i g h t)$ with the chemical evolution model of Galliano et al. (2008). The colours and symbols are the same as for Fig.4. The dark grey stripes show the range of values from the Galliano et al. (2008) chemical evolution model. The black dashed line represents the reference scaling of the G/D with metallicity (not fit to the data). The black dotted and dash-dotted lines represent the best power-law and best broken power-law fits to the data.

strong simplifying assumption made by Galliano et al. (2008) is that they did not take into account dust growth in the ISM as they assume full condensation of the grains. In the Galaxy, the typical timescale for dust formation by stars has been shown to be larger than the typical timescale for dust destruction (Jones \& Tielens 1994; Jones et al. 1996). Because we still observe dust in the ISM, we need to reach equilibrium between formation and destruction of the dust grains, either via high $\mathrm{SN}$ yields or dust growth processes in the ISM. In the following sections we thus look at models including dust growth in the ISM.

\subsection{Including dust growth in the ISM}

Asano et al. (2013a) propose a chemical evolution model, based on models from Hirashita (1999) and Inoue (2011), taking into account the evolution of the metal content in the dust phase in addition to the evolution of the total amount of metals. The dust formation is regulated by asymptotic giant branch (AGB) stars, SNII and dust growth, via accretion, in the ISM. The dust is destroyed by SN shocks. Inflows and outflows are not considered (closed-box model) and the total mass of the galaxy is constant and set to $10^{10} M_{\odot}$. Metallicity and age dependence of the various dust formation processes are taken into account. Asano et al. (2013a) show that dust growth in the ISM becomes the main driver of the dust mass evolution, compared to the dust formation from metals produced and ejected into the ISM by stars, when the metallicity of the galaxy exceeds a certain "critical" metallicity. This critical metallicity increases with decreasing star formation timescale ${ }^{9}$. Asano et al. (2013a) show that dust growth via accretion processes in the ISM is regulated by this critical metallicity over a large range of star formation timescales (for $\left.\tau_{\mathrm{SF}}=0.5-5-50 \mathrm{Gyr}\right)$. After reaching this critical metallicity the dust mass increases more rapidly, boosted by dust growth processes, before saturating when all of the metals available for dust formation are locked up in dust. The metallicity at which

\footnotetext{
9 The star formation timescale, $\tau_{\mathrm{SF}}$, is defined by the timescale during which star formation occurs: $\tau_{\mathrm{SF}}=\left(M_{\mathrm{ISM}}\right) / S F R$, where SFR is the star formation rate (see Eq. (5) of Asano et al. 2013a). This is not to be mixed with the timescale, $\tau$, in exponentially decaying star formation histories going as $\exp ^{-(t / \tau)}$.
}

this saturation occurs thus also depends on the critical metallicity, which in turn depends on the star formation history of the galaxy.

Figure 7 shows the models of Asano et al. (2013a) (for $\tau_{\mathrm{SF}}=0.5-5-50$ Gyr) overlaid on the observed G/D values. The models were originally on an arbitrary scale and they are thus normalised at the $(G / D))_{\odot}$ value. We assume an error on this value of $\sim 60 \%$, from the range of values determined from the fits in Sect. 3.3, to have a tolerance range around the model (shown by the shaded grey area in Fig. 7). The three models show similar evolution with metallicity and indeed are homologous to each other when normalised by their respective critical metallicities (see Fig. 3 of Asano et al. 2013a). We clearly see the influence of the critical metallicity on the dust mass evolution: at low metallicities the range of possible $\mathrm{G} / \mathrm{D}$ values (illustrated by the grey area in Fig. 7) becomes wider around $12+\log (\mathrm{O} / \mathrm{H}) \sim 7.2-7.3$ before narrowing down around $12+\log (\mathrm{O} / \mathrm{H}) \sim 8.6$. This broadening is due to the fact that in this range of metallicities, galaxies with high star formation timescales have already reached their critical metallicity and have a rapidly increasing dust mass (and thus a low G/D at a given metallicity), compared to galaxies with lower star formation timescales which have not yet reached this critical metallicity and with a dust mass still regulated by stars (thus with a higher G/D at the same metallicity). Galaxies with high star formation timescales then reach saturation at moderate metallicities as they started their "active dust growth" phase at a lower critical metallicity (i.e., earlier in their evolution), while, at the same metallicity, galaxies with low star formation timescales are still in the "active dust growth" phase. Then when these galaxies also reach saturation, because the dust growth in the ISM becomes ineffective, the range of possible $\mathrm{G} / \mathrm{D}$ values narrows down.

From Fig. 7 we see that the models from Asano et al. (2013a) are consistent with the G/D from both $X_{\mathrm{CO}}$ values. We note that below $12+\log (\mathrm{O} / \mathrm{H}) \sim 7.5$, even the $\tau_{\mathrm{SF}}=50$ Gyr model does not agree anymore with the reference scaling of the G/D with metallicity (and below $12+\log (\mathrm{O} / \mathrm{H}) \sim 8.0$ for $\tau_{\mathrm{SF}}=5 \mathrm{Gyr}$ ). The other two empirical relations (our best power-law and broken power-law fits) are consistent with the models of Asano et al. (2013a) within the considered metallicity range: from $12+\log (\mathrm{O} / \mathrm{H}) \sim 7.0$ to 9.1 . Comparing with the shape of the 

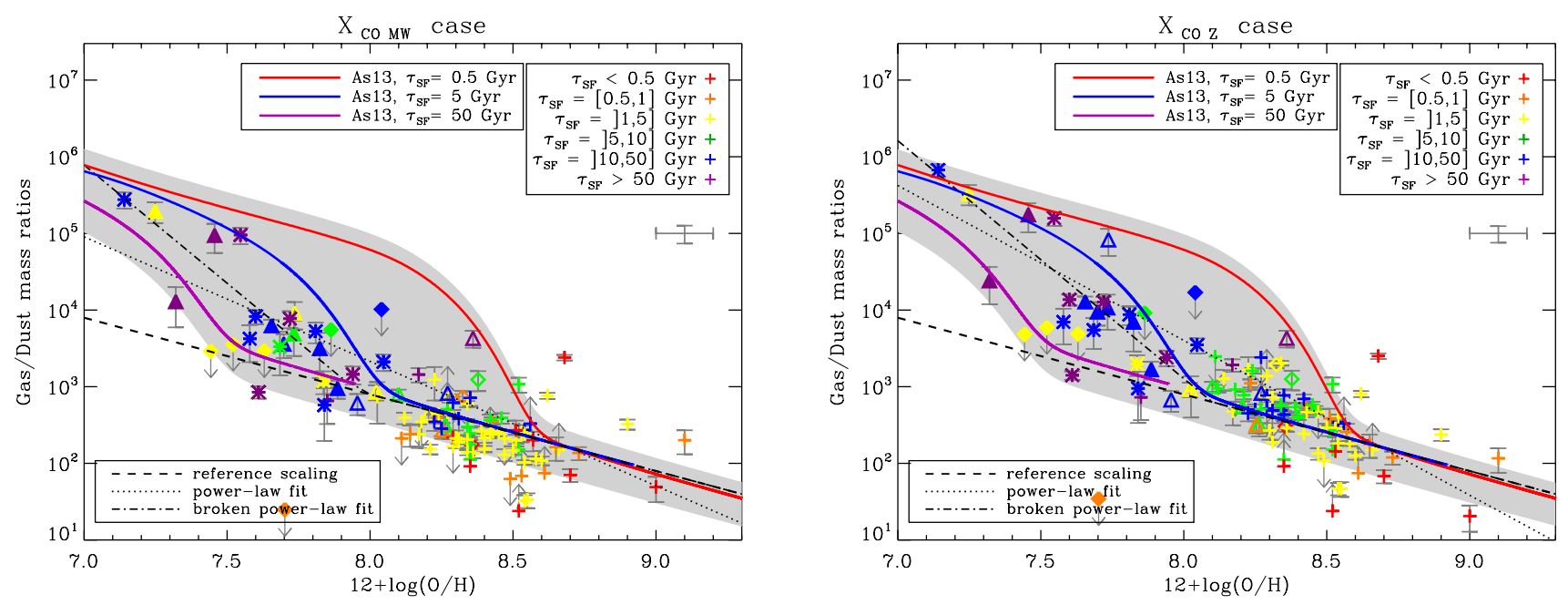

Fig. 7. G/D as a function of metallicity for the 2 values of $X_{\mathrm{CO}}: X_{\mathrm{CO}, \mathrm{MW}}(l e f t)$ and $X_{\mathrm{CO}, Z}($ right $)$ with the chemical evolution model of Asano et al. (2013a). The symbols are the same as for Fig. 4. The colours delineate ranges in star formation timescales $\tau_{\mathrm{SF}}$. The model from Asano et al. (2013a) is overlaid on the points for various $\tau_{\mathrm{SF}}=0.5$ (red), 5 (blue), 50 (purple) Gyr. The black dashed line represents the reference scaling of the G/D with metallicity (not fit to the data). The black dotted and dash-dotted lines represent the best power-law and best broken power-law fits to the data.

Asano et al. (2013a) models, our best broken power-law fit may overestimate the $\mathrm{G} / \mathrm{D}$ for $12+\log (\mathrm{O} / \mathrm{H}) \leq 7.0$. However, Izotov et al. (2012) recently suggested that there seems to be a metallicity floor around $12+\log (\mathrm{O} / \mathrm{H}) \sim 6.9$, below which no galaxies are found in the local Universe, as already proposed by Kunth \& Sargent (1986). Thus our metallicity range is close to being the largest achievable in the local Universe as far as low metallicities are concerned.

The galaxies from the DGS, KINGFISH and G11 samples are colour coded in Fig. 7 by an approximation of their star formation timescale $\tau_{\mathrm{SF}}$, estimated from $\tau_{\mathrm{SF}}=\left(M_{\mathrm{gas}}+M_{\mathrm{dust}}\right) / S F R$, where the SFR have been estimated from $L_{\mathrm{TIR}}$ (obtained by integrating over the modelled SEDs between 1 and $1000 \mu \mathrm{m}$, Rémy-Ruyer et al., in prep.). The $\tau_{\mathrm{SF}}$ values are roughly consistent with the models from Asano et al. (2013a). The median value of $\tau_{\mathrm{SF}}$ is $\sim 3.0\left(X_{\mathrm{CO}, \mathrm{MW}}\right)$ and $5.5\left(X_{\mathrm{CO}, \mathrm{Z}}\right) \mathrm{Gyr}$, but with a large dispersion of $\sim 20 \mathrm{Gyr}$ around this value. As the models from Asano et al. (2013a) encompass most of the observed G/D values, the dispersion seen in the $G / D$ values can be due to the wide range of star formation timescales in the considered galaxies. This is consistent with the large dispersion in the approximated star formation timescales in our sample.

In Asano et al. (2013a), the star formation is assumed to be continuous over the star formation timescale. However, star formation histories of many dwarf galaxies derived from colourmagnitude diagrams reconstruction show distinct episodes of star formation separated by more quiescent phases (e.g., Tolstoy et al. 2009, and references therein). For example, Legrand et al. (2000) suggested for IZw18 a star formation history made of bursts of star formation in between more quiescent phases, following the suggestion of Searle \& Sargent (1972). Episodic star formation histories have also been suggested in Nbody/smoothed particle hydrodynamics simulations of dwarf galaxy evolution (e.g., Valcke et al. 2008; Revaz \& Jablonka 2012). As we saw that the scatter in Fig. 7 seems to be due to the range of star formation timescales probed by our sample, and assuming a continuous star formation, we thus need to consider the influence of the continuous vs. episodic star formation modes.

\subsection{Episodic versus continuous star formation}

In the following we compare the observationally derived G/D with results of dust evolution models in dwarf galaxies with episodic star formation history from Zhukovska (2014). These models were originally introduced to study the lifecycle of dust species from different origins in the Solar neighbourhood (Zhukovska et al. 2008). The model of Zhukovska (2014) is based on the Zhukovska et al. (2008) model that has been adapted to treat dwarf galaxies, specifically by considering episodic star formation. In Zhukovska (2014), the equations describing the evolution of the galaxy are now normalised to the total galactic masses $M_{\text {tot }}$ (instead of surface densities) because dwarf galaxies are smaller in size and thus assumed to have a well-mixed ISM. As in Zhukovska et al. (2008), the modelled dwarf galaxy is formed by gas infall starting from $M_{\text {tot }}=0$ and reaches its total mass $M_{\mathrm{tot}}$ on the infall timescale. The assumed value of the infall timescale in Zhukovska (2014) is set to a much shorter value for dwarf galaxies than for the Solar neighbourhood model. Since the G/D is the ratio of the gas and dust masses, it does not depend on the normalisation by the total mass $M_{\text {tot }}$, and is determined by the star formation history and infall timescale. We refer the reader to Zhukovska (2014), for more details on the modelling.

Similarly to the models from Asano et al. (2013a), Zhukovska (2014) include dust formation in AGB stars, SN II and dust growth by mantle accretion in the ISM. The main difference between these models resides in the treatment of dust growth. Zhukovska (2014) assume a two-phase ISM consisting of clouds and an intercloud medium, where clouds are characterised by temperature, density, mass fraction, and lifetime. Dust growth by accretion in their model takes place only in the dense gas and also critically depends on the metallicity (see Zhukovska 2008).

In this paper, we consider three models from Zhukovska (2014), which differ only in duration and intensity of the star formation bursts. All of the models consider six bursts of star formation starting at instants $t=0.5,1,2,5,7$, and $11 \mathrm{Gyr}$. In the first and the second model, the burst duration is $50 \mathrm{Myr}$ and $500 \mathrm{Myr}$, respectively, and the $\tau_{\mathrm{SF}}$ during bursts is 2 Gyr. The 

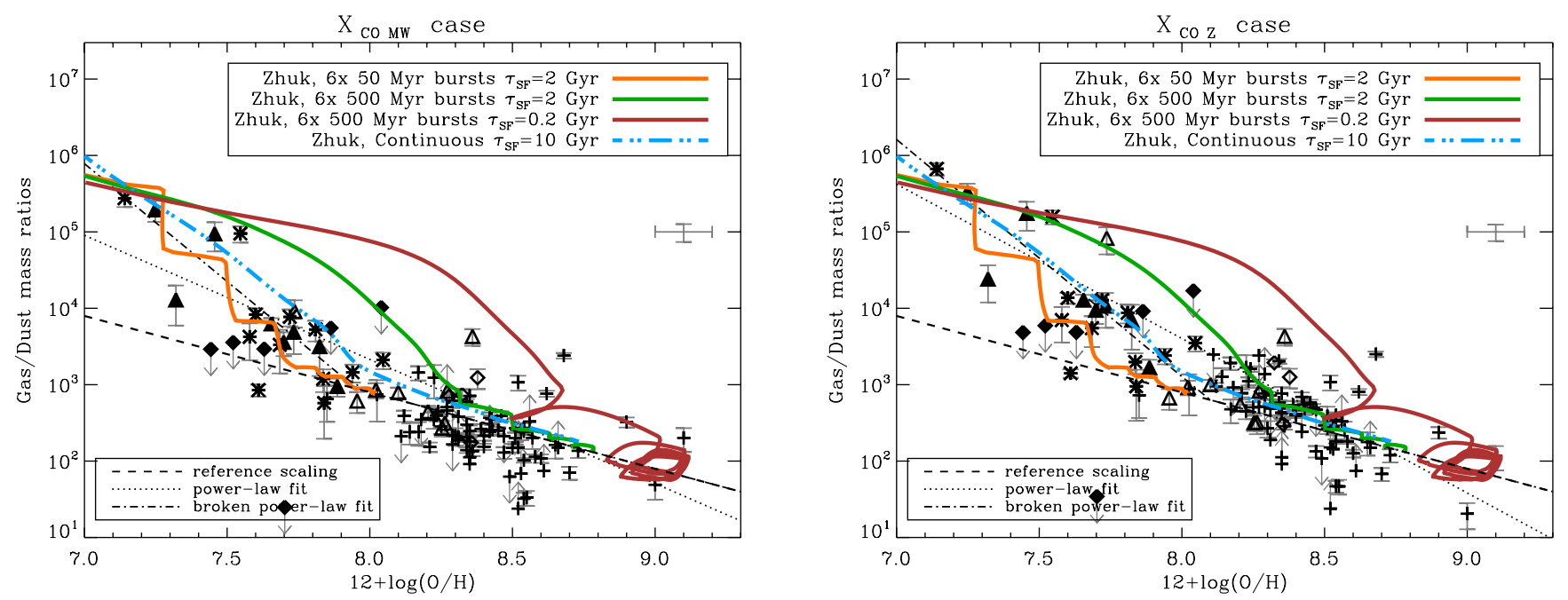

Fig. 8. G/D as a function of metallicity for the 2 values of $X_{\mathrm{CO}}: X_{\mathrm{CO}, \mathrm{MW}}($ left $)$ and $X_{\mathrm{CO}, Z}($ right $)$ with the chemical evolution model of Zhukovska (2014). The symbols are the same as for Fig. 4. The model from Zhukovska (2014) is shown for various star formation histories: episodic with 6 bursts of $50 \mathrm{Myr}$ and star formation timescale of $\tau_{\mathrm{SF}}=2 \mathrm{Gyr}$ (orange), episodic with 6 bursts of $500 \mathrm{Myr}$ and $\tau_{\mathrm{SF}}=2 \mathrm{Gyr}$ (green), episodic with 6 bursts of $500 \mathrm{Myr}$ and $\tau_{\mathrm{SF}}=0.2 \mathrm{Gyr}$ (more intense star formation, brown) and continuous with a star formation timescale $\tau_{\mathrm{SF}}=10 \mathrm{Gyr}$ (cyan dash-3 dots line). The black dashed line represents the reference scaling of the G/D with metallicity (not fit to the data). The black dotted and dash-dotted lines represent the best power-law and best broken power-law fits to the data.

second model is typical of a low-metallicity dwarf galaxy. In the third model, the burst duration is $500 \mathrm{Myr}$ but the value of $\tau_{\mathrm{SF}}$ is much shorter, 0.2 Gyr. During the quiescence phases $\tau_{\mathrm{SF}}$ is set to be 200 Gyr. We also consider a model with continuous star formation on a $10 \mathrm{Gyr}$ timescale, for comparison. In all of the models, the infall timescale is $0.3 \mathrm{Gyr}$ and there are no galactic outflows. The initial metallicity of the infalling gas is set to be $10^{-4}$ with SNII like enhanced $[\alpha / \mathrm{Fe}]$ ratio.

The models from Zhukovska (2014) are presented in Fig. 8 and reproduce the broadening of the observed $\mathrm{G} / \mathrm{D}$ values at low metallicities $(12+\log (\mathrm{O} / \mathrm{H}) \lesssim 8.3)$, and also converge around $12+\log (\mathrm{O} / \mathrm{H}) \sim 7.2$, similar to the models of continuous star formation of Asano et al. (2013a). Note how the star formation history impacts the shape of the modelled G/D: the most extreme $\mathrm{G} / \mathrm{D}$ values are obtained by the three models with episodic bursts of star formation. For the model with more intense star formation (brown curve in Fig. 8), $12+\log (\mathrm{O} / \mathrm{H})=8.6$ is reached during the first burst, and very high values of the G/D are quickly reached, up to two orders of magnitude above the reference scaling relation at moderate metallicities $(12+\log (\mathrm{O} / \mathrm{H}) \sim 8.2-$ 8.3). It also presents an interesting scatter of $\mathrm{G} / \mathrm{D}$ values near $12+\log (\mathrm{O} / \mathrm{H})=9.0$ that is due to dust destruction during the SF bursts, and consistent with the scatter predicted by the Galliano et al. (2008) model (see Fig. 9). The fact that the lowmetallicity slope of the broken power law is consistent with the continuous star formation model at low metallicities for the $X_{\mathrm{CO}, Z}$ case indicates that this broken power law can provide a fairly good empirical way of estimating the G/D for a given metallicity.

\subsection{Explaining the observed scatter in $G / D$ values}

Figure 9 shows the three models overlaid on the observed G/D values. The models from Asano et al. (2013a) and Zhukovska (2014) provide trends that are consistent with each other and with the data and its scatter. More dust observations of extremely low-metallicity galaxies with $12+\log (\mathrm{O} / \mathrm{H})<7.5$ are nonetheless needed to confirm this agreement between the models and the data at very low metallicities. The model of Galliano et al. (2008) fails to reproduce the observed G/D at low metallicities, but provides a good complement to explain the scatter seen at high metallicities, consistent with the predictions of the third bursty model by Zhukovska (2014). We thus conclude that the observed scatter at low metallicities in the G/D values is due to the wide variety of environments we are probing, and especially to the different star formation histories. The observed scatter at higher metallicity seems to be due to different timescales for dust destruction by SN blast waves in the different environments and to the efficiency of dust shattering in the ISM.

We investigated here two different parameters to explain the scatter in the G/D values: star formation histories and efficiency of dust destruction, but other processes could also give rise to the observed scatter. In our dust modelling we allow the mass fraction of small grains compared to big grains to vary from galaxy to galaxy (controlled by the $f_{\mathrm{vsg}}$ parameter), to account for potential variations in the grain size distribution. This had already been done in one low-metallicity galaxy by Lisenfeld et al. (2002). On the theoretical side, Hirashita \& Kuo (2011) showed that the dust grain size distribution can have an important impact on the dust growth process in the ISM by regulating the grain growth rate. They also showed that the critical metallicity mentioned in Sect. 4.2, for which grain growth becomes dominant, also depends on the grain size distribution. Additionally the grain size distribution varies as the galaxy evolves and this evolution is controlled by different dust formation processes at different ages (Asano et al. 2013b). Thus the observed scatter can also be due to variations of the grain size distribution between the galaxies, the effect of which can be related to the star formation history.

As discussed in Sect. 3.4, we use a dust model with the dust composition and optical properties representative of dust in the Milky Way for all of the galaxies. Another explanation for the scatter seen at all metallicities could be that the dust composition in fact varies between the galaxies, leading to large variations in the emissivity of the dust grains (Jones 2012). This would then imply dust masses relatively similar at a given metallicity but large variations in the emissivity properties of dust from one galaxy to another. With our fixed emissivity (due to our fixed dust composition in our dust model) this effect would be seen 

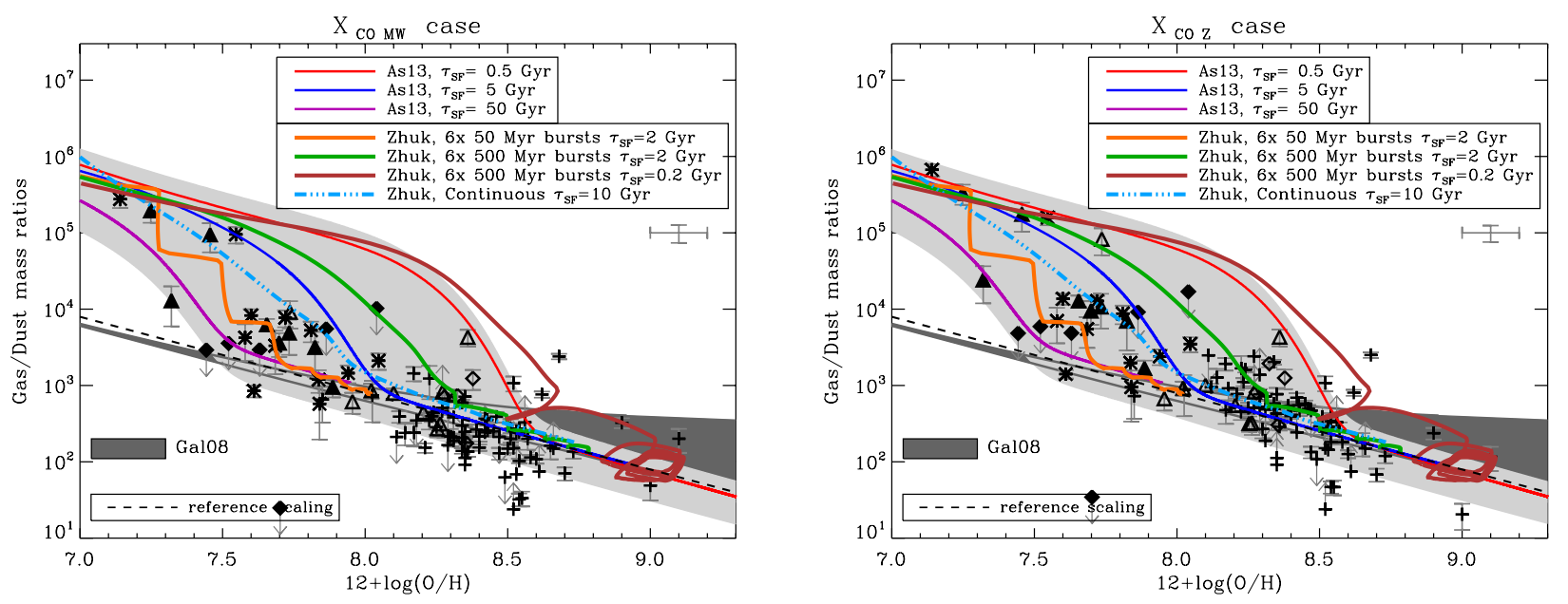

Fig. 9. G/D as a function of metallicity for the 2 values of $X_{\mathrm{CO}}: X_{\mathrm{CO}, \mathrm{MW}}(l e f t)$ and $X_{\mathrm{CO}, Z}(r i g h t)$ with the three chemical evolution model considered in Sect. 4. The symbols are the same as for Fig. 4. The model ranges from Galliano et al. (2008) are delineated by the dark grey stripe. The models from Asano et al. (2013a) are shown with the red, blue and purple lines and the light grey tolerance zone. The models from Zhukovska (2014) are shown with the orange, green brown solid lines and cyan dash-3 dots line. The black dashed line represents the reference scaling of the G/D with metallicity (not fit to the data).

through the variations in the derived dust masses (and then in the $\mathrm{G} / \mathrm{D}$ values) seen at a given metallicity, thus giving rise to the observed scatter.

Another aspect not taken into account in the chemical models we use here is that the mass of the galaxy plays an important role in its chemical evolution. According to the chemical downsizing scenario, galaxies with different masses have different star formation efficiencies (e.g., Brooks et al. 2007). The massive galaxies form stars before low-mass galaxies in the history of the Universe (Cowie et al. 1996). The exact reasons are not well known, some models would argue that this is a feedback effect, with metals being lost in outflows in less massive galaxies (Frye et al. 2002), and others would argue that the local density and the ISM pressure is responsible (e.g., Cen \& Ostriker 1999): if the ISM density is systematically lower in a dwarf galaxy, for instance, then the star formation activity will be low on average, impacting the G/D value. A massive galaxy will not show the same behaviour, and this difference will also introduce some scatter in the observed G/D at a given metallicity.

Additionally external processes such as outflows or interactions and mergers, not considered here either in our models, can also be responsible for the scatter in the G/D values.

\subsection{Implications for the observed $G / D$ in galaxies}

We saw in Sects. 4.2 and 4.3 that the broken power-law relation in the $X_{\mathrm{CO}, Z}$ case is consistent with the predictions from both chemical evolution models. Even though there are only five galaxies with $12+\log (\mathrm{O} / \mathrm{H}) \sim 7.5$ to constrain the observed $\mathrm{G} / \mathrm{D}$ at extremely low metallicities, this broken power-law relation is the best empirical estimate of the observed G/D for local galaxies we have at our disposal so far. Thus, we advise to use this empirical prescription to estimate the G/D based on a metallicity value for local galaxies, though keeping in mind the large scatter in the observed G/D values and the uncertainties on the broken power-law parameters: the estimated G/D would be accurate to a factor of $\sim 1.6$. Note also that this empirical relation has been derived for metallicities estimated with a strong emission line method and the calibration from Pilyugin \& Thuan (2005). Thus any estimation of the G/D with this empirical relation should be done from a metallicity derived with the same method. Additionally, more observations of the dust in extremely lowmetallicity galaxies are needed in order to place more constraints on this empirical relation at extremely low metallicities.

\section{Conclusions}

We present here the evolution of gas-to-dust mass ratios for a sample of 126 galaxies over a 2 dex metallicity range: from $12+\log (\mathrm{O} / \mathrm{H})=7.14$ to 9.10 . We use two different values for the $X_{\mathrm{CO}}$ factor: the Galactic value, $X_{\mathrm{CO}, \mathrm{MW}}$, and a metallicity dependent value, $X_{\mathrm{CO}, Z}\left(\propto Z^{-2}\right)$, to determine the $\mathrm{G} / \mathrm{D}$ relation with metallicity. We consider several empirical trends to describe the G/D as a function of metallicity: power law with a slope fixed to -1 ("reference" relation) and a slope left free, and a broken power law. We also compare the observed G/D evolution with metallicity with predictions from chemical evolution models from Galliano et al. (2008); Asano et al. (2013a) and Zhukovska (2014). We show that:

- The G/D correlates with metallicity, stellar mass, SFR and SSFR. However, the correlation is weaker with the last three parameters, which themselves depend on metallicity. Thus metallicity is the main physical property of the galaxy driving the observed $\mathrm{G} / \mathrm{D}$ values.

- The observed G/D vs metallicity relation cannot be represented by a power law with a slope of -1 at all metallicities. The observed trend is steeper at low metallicities and could be due to the harder ISRF in low metallicity dwarf galaxies that affects the balance between dust formation and destruction by limiting the accretion or enhancing the destruction of the dust grains.

- There is a large scatter in the G/D values for a given metallicity throughout the metallicity range: in metallicity bins of $\sim 0.1 \mathrm{dex}$, the dispersion is $\sim 0.37$ dex on average for all bins and for both $X_{\mathrm{CO}}$ values. This scatter does not depend on the metallicity indicating that metallicity may not the main driver of the observed scatter.

- A power-law fit to the data gives a slope $\alpha$ of $-1.6 \pm$ $0.3\left(X_{\mathrm{CO}, \mathrm{Mw}}\right)$ or $-2.0 \pm 0.3\left(X_{\mathrm{CO}, \mathrm{Z}}\right)$. A broken power-law fit, with a fixed high-metallicity slope $\alpha_{\mathrm{H}}=-1$, gives 
a low-metallicity slope, $\alpha_{\mathrm{L}}$, of $-3.1 \pm 1.8\left(X_{\mathrm{CO}, \mathrm{MW}}\right)$ or $-3.1 \pm 1.3\left(X_{\mathrm{CO}, Z}\right)$, with a transition metallicity of $7.96 \pm$ $0.47\left(X_{\mathrm{CO}, \mathrm{MW}}\right)$ or $8.10 \pm 0.43\left(X_{\mathrm{CO}, Z}\right)$. If left free, the highmetallicity slope is consistent within errors with the slope of -1 observed by other studies. On average, the broken power law reproduces best the observed G/D compared to the two power laws ( $\alpha=-1$ or free) and provides estimates of the $\mathrm{G} / \mathrm{D}$ that are accurate to a factor of 1.6.

- We recommend using the best broken power-law fit from the $X_{\mathrm{CO}, Z}$ case to empirically estimate a G/D from a metallicity, though keeping in mind the large uncertainty on the estimated G/D value, and the differences existing between the different methods to estimate metallicities.

- Chemical evolution models from Asano et al. (2013a) (including dust growth in the ISM as a source of dust production and continuous star formation) and Zhukovska (2014) (testing episodic star formation histories and effects of dust growth), are consistent with the observed G/D trend with metallicity for both $X_{\mathrm{CO}}$ values, implying that dust growth in the ISM and the global star formation history are both important in the evolution of the G/D. The model from Galliano et al. (2008), while it well reproduces the observations at higher metallicity, does not reproduce the observed trend at low metallicities because of too-simple assumptions in their model. The three models confirm that the reference scaling of the G/D with metallicity is not plausible at low metallicities below $12+\log (\mathrm{O} / \mathrm{H}) \sim 7.7$. Our best power-law fit with a free slope, and broken power-law fit are consistent with the Asano et al. (2013a) and Zhukovska (2014) models.

- The scatter present at all metallicities is due to the variety of the considered environments, and is consistent with predictions of models considered for this study. Variation in the star formation histories of the galaxies and in the dust destruction efficiency are explored with the different models, and can explain the observed scatter in the G/D values at all metallicities. Variations in the dust properties: grain size distribution and chemical composition can also be invoked, as well as interactions of the galaxies with their surroundings. However, all of these effects are highly affected by numerous degeneracies, which cannot be disentangled at this stage.

Acknowledgements. The authors would like to thank the anonymous referee for his/her helpful comments. The authors would like to thank H. Hirashita for interesting discussions on the chemical evolution models. A.R.R. is supported by a CFR grant from the AIM laboratory (Saclay, France). This research was, in part, made possible through the financial support of the Agence Nationale de la Recherche (ANR) through the programme SYMPATICO (Program Blanc Projet ANR-11-BS56-0023). S.Z. acknowledges support by the Deutsche Forschungsgemeinschaft through SPP 1573: "Physics of the Interstellar Medium". I.D.L. is a postdoctoral researcher of the FWO-Vlaanderen (Belgium). PACS has been developed by MPE (Germany); UVIE (Austria); KU Leuven, CSL, IMEC (Belgium); CEA, LAM (France); MPIA (Germany); INAFIFSI/OAA/OAP/OAT, LENS, SISSA (Italy); IAC (Spain). This development has been supported by BMVIT (Austria), ESA-PRODEX (Belgium), CEA/CNES (France), DLR (Germany), ASI/INAF (Italy), and CICYT/MCYT (Spain). SPIRE has been developed by Cardiff University (UK); Univ. Lethbridge (Canada); NAOC (China); CEA, LAM (France); IFSI, Univ. Padua (Italy); IAC (Spain); SNSB (Sweden); Imperial College London, RAL, UCL-MSSL, UKATC, Univ. Sussex (UK) and Caltech, JPL, NHSC, Univ. Colorado (USA). This development has been supported by CSA (Canada); NAOC (China); CEA, CNES, CNRS (France); ASI (Italy); MCINN (Spain); Stockholm Observatory (Sweden); STFC (UK); and NASA (USA). SPIRE has been developed by a consortium of institutes led by Cardiff Univ. (UK) and including: Univ. Lethbridge (Canada); NAOC (China); CEA, LAM (France); IFSI, Univ. Padua (Italy); IAC (Spain); Stockholm Observatory (Sweden); Imperial College London, RAL, UCL-MSSL, UKATC, Univ. Sussex (UK); and Caltech, JPL, NHSC, Univ. Colorado (USA). This development has been supported by national funding agencies: CSA (Canada); NAOC (China); CEA, CNES, CNRS (France); ASI (Italy); MCINN (Spain); SNSB (Sweden); STFC, UKSA (UK); and NASA (USA).

\section{References}

Ackermann, M., Ajello, M., Baldini, L., et al. 2011, ApJ, 726, 81

Albrecht, M., Chini, R., Krügel, E., Müller, S. A. H., \& Lemke, R. 2004, A\&A, 414,141

Asano, R. S., Takeuchi, T. T., Hirashita, H., \& Inoue, A. K. 2013a, Earth Planets Space, 65, 213

Asano, R. S., Takeuchi, T. T., Hirashita, H., \& Nozawa, T. 2013b, MNRAS, 432, 637

Asplund, M., Grevesse, N., Sauval, A. J., \& Scott, P. 2009, ARA\&A, 47, 481

Begum, A., \& Chengalur, J. N. 2005, MNRAS, 362, 609

Bendo, G. J., Dale, D. A., Draine, B. T., et al. 2006, ApJ, 652, 283

Bendo, G. J., Wilson, C. D., Warren, B. E., et al. 2010, MNRAS, 402, 1409

Bendo, G. J., Galliano, F., \& Madden, S. C. 2012, MNRAS, 423, 197

Bernard, J.-P., Reach, W. T., Paradis, D., et al. 2008, AJ, 136, 919

Bettoni, D., Galletta, G., \& García-Burillo, S. 2003, A\&A, 405, 5

Bianchi, S. 2013, A\&A, 552, A89

Bocchio, M., Micelotta, E. R., Gautier, A.-L., \& Jones, A. P. 2012, A\&A, 545, A124

Bocchio, M., Jones, A. P., Verstraete, L., et al. 2013, A\&A, 556, A6

Bolatto, A. D., Wolfire, M., \& Leroy, A. K. 2013, ARA\&A, 51, 207

Bonatto, C. J., \& Pastoriza, M. G. 1990, ApJ, 353, 445

Boselli, A., Lequeux, J., \& Gavazzi, G. 2002, A\&A, 384, 33

Boselli, A., Eales, S., Cortese, L., et al. 2010, PASP, 122, 261

Bot, C., Ysard, N., Paradis, D., et al. 2010, A\&A, 523, A20

Böttner, C., Klein, U., \& Heithausen, A. 2003, A\&A, 408, 493

Brooks, A. M., Governato, F., Booth, C. M., et al. 2007, ApJ, 655, L17

Cannon, J. M., McClure-Griffiths, N. M., Skillman, E. D., \& Côté, S. 2004, ApJ, 607,274

Carignan, C., \& Beaulieu, S. 1989, ApJ, 347, 760

Carignan, C., Beaulieu, S., \& Freeman, K. C. 1990, AJ, 99, 178

Cen, R., \& Ostriker, J. P. 1999, ApJ, 519, L109

Chengalur, J. N., Pustilnik, S. A., Martin, J.-M., \& Kniazev, A. Y. 2006 MNRAS, 371, 1849

Cormier, D., Madden, S. C., Hony, S., et al. 2010, A\&A, 518, L57

Cormier, D., Lebouteiller, V., Madden, S. C., et al. 2012, A\&A, 548, A20

Cormier, D., Madden, S. C., Lebouteiller, V., et al. 2014, A\&A, accepted [arXiv: 1401.0563]

Cowie, L. L., Songaila, A., Hu, E. M., \& Cohen, J. G. 1996, AJ, 112, 839

Dahlem, M., Weaver, K. A., \& Heckman, T. M. 1998, ApJS, 118, 401

Dale, D. A., Helou, G., Contursi, A., Silbermann, N. A., \& Kolhatkar, S. 2001a ApJ, 549, 215

Dale, D. A., Helou, G., Neugebauer, G., et al. 2001b, AJ, 122, 1736

Dale, D. A., Gil de Paz, A., Gordon, K. D., et al. 2007, ApJ, 655, 863

Dale, D. A., Aniano, G., Engelbracht, C. W., et al. 2012, ApJ, 745, 95

Davoust, E., \& Contini, T. 2004, A\&A, 416, 515

de Blok, W. J. G., \& Walter, F. 2006, AJ, 131, 343

Di Matteo, P., Montuori, M., Lehnert, M. D., Combes, F., \& Semelin, B. 2011,

in IAU Symp. 277, eds. C. Carignan, F. Combes, \& K. C. Freeman, 246

Draine, B. T., \& Hensley, B. 2012, ApJ, 757, 103

Draine, B. T., \& Li, A. 2007, ApJ, 657, 810

Draine, B. T., Dale, D. A., Bendo, G., et al. 2007, ApJ, 663, 866

Dumke, M., Krause, M., \& Wielebinski, R. 2004, A\&A, 414, 475

Dwek, E. 1998, ApJ, 501, 643

Dwek, E., \& Scalo, J. M. 1980, ApJ, 239, 193

Ekta, B., \& Chengalur, J. N. 2010, MNRAS, 403, 295

Ekta, B., Pustilnik, S. A., \& Chengalur, J. N. 2009, MNRAS, 397, 963

Engelbracht, C. W., Gordon, K. D., Rieke, G. H., et al. 2005, ApJ, 628, L29

Engelbracht, C. W., Rieke, G. H., Gordon, K. D., et al. 2008, ApJ, 678, 804

Eskew, M., Zaritsky, D., \& Meidt, S. 2012, AJ, 143, 139

Frye, B., Broadhurst, T., \& Benítez, N. 2002, ApJ, 568, 558

Galametz, M., Madden, S., Galliano, F., et al. 2009, A\&A, 508, 645

Galametz, M., Madden, S. C., Galliano, F., et al. 2011, A\&A, 532, A56

Galametz, M., Hony, S., Galliano, F., et al. 2013, MNRAS, 431, 1596

Galliano, F., Madden, S. C., Jones, A. P., et al. 2003, A\&A, 407, 159

Galliano, F., Madden, S. C., Jones, A. P., Wilson, C. D., \& Bernard, J.-P. 2005 A\&A, 434, 867

Galliano, F., Dwek, E., \& Chanial, P. 2008, ApJ, 672, 214

Galliano, F., Hony, S., Bernard, J.-P., et al. 2011, A\&A, 536, A88

Garnett, D. R., Kennicutt, Jr., R. C., \& Bresolin, F. 2004, ApJ, 607, L21

Gil de Paz, A., Madore, B. F., \& Pevunova, O. 2003, ApJS, 147, 29

Gordon, D., \& Gottesman, S. T. 1981, AJ, 86, 161

Gratier, P., Braine, J., Rodriguez-Fernandez, N. J., et al. 2010, A\&A, 512, A68

Greve, A., Becker, R., Johansson, L. E. B., \& McKeith, C. D. 1996, A\&A, 312, 391

Grossi, M., Hunt, L. K., Madden, S., et al. 2010, A\&A, 518, L52

Guelin, M., Zylka, R., Mezger, P. G., et al. 1993, A\&A, 279, L37 
Guelin, M., Zylka, R., Mezger, P. G., Haslam, C. G. T., \& Kreysa, E. 1995, A\&A, 298, L29

Hirashita, H. 1999, ApJ, 522, 220

Hirashita, H., \& Kuo, T.-M. 2011, MNRAS, 416, 1340

Hirashita, H., Tajiri, Y. Y., \& Kamaya, H. 2002, A\&A, 388, 439

Hony, S., Galliano, F., Madden, S. M., \& SAGE Consortium 2010, BAAS, 42, 487

Huchtmeier, W. K. 1979, A\&A, 75, 170

Huchtmeier, W. K., \& Richter, O.-G. 1988, A\&A, 203, 237

Huchtmeier, W. K., Seiradakis, J. H., \& Materne, J. 1981, A\&A, 102, 134

Huchtmeier, W. K., Krishna, G., \& Petrosian, A. 2005, A\&A, 434, 887

Hunt, L., Bianchi, S., \& Maiolino, R. 2005, A\&A, 434, 849

Hunter, D. 1997, PASP, 109, 937

Hunter, D. A., van Woerden, H., \& Gallagher, J. S. 1999, AJ, 118, 2184

Hunter, D. A., Zahedy, F., Bowsher, E. C., et al. 2011, AJ, 142, 173

Inoue, A. K. 2011, Earth Planets Space, 63, 1027

Israel, F. P. 1997, A\&A, 328, 471

Israel, F. P. 2005, A\&A, 438, 855

Israel, F. P., Maloney, P. R., Geis, N., et al. 1996, ApJ, 465, 738

Israel, F. P., Baas, F., Rudy, R. J., Skillman, E. D., \& Woodward, C. E. 2003, A\&A, 397, 87

Issa, M. R., MacLaren, I., \& Wolfendale, A. W. 1990, A\&A, 236, 237

Izotov, Y. I., Thuan, T. X., \& Guseva, N. G. 2012, A\&A, 546, A122

James, A., Dunne, L., Eales, S., \& Edmunds, M. G. 2002, MNRAS, 335, 753

Jones, A. P. 2012, A\&A, 545, C3

Jones, A., \& Tielens, A. 1994, in The Cold Universe, eds. T. Montmerle, C. J. Lada, I. F. Mirabel, \& J. Tran Thanh Van, 35

Jones, A. P., Tielens, A. G. G. M., Hollenbach, D. J., \& McKee, C. F. 1994, ApJ, 433, 797

Jones, A. P., Tielens, A. G. G. M., \& Hollenbach, D. J. 1996, ApJ, 469, 740

Karczewski, O. Ł., Barlow, M. J., Page, M. J., et al. 2013, MNRAS, 431, 2493

Kennicutt, Jr., R. C. 1998, ApJ, 498, 541

Kennicutt, Jr., R. C., Bresolin, F., \& Garnett, D. R. 2003, ApJ, 591, 801

Kennicutt, Jr., R. C., Hao, C.-N., Calzetti, D., et al. 2009, ApJ, 703, 1672

Kennicutt, R. C., Calzetti, D., Aniano, G., et al. 2011, PASP, 123, 1347

Kewley, L. J., \& Ellison, S. L. 2008, ApJ, 681, 1183

Kobulnicky, H. A., Dickey, J. M., Sargent, A. I., Hogg, D. E., \& Conti, P. S. 1995, AJ, 110, 116

Kunth, D., \& Sargent, W. L. W. 1986, ApJ, 300, 496

Lee, J. C., Salzer, J. J., Impey, C., Thuan, T. X., \& Gronwall, C. 2002, AJ, 124, 3088

Legrand, F., Kunth, D., Roy, J.-R., Mas-Hesse, J. M., \& Walsh, J. R. 2000, A\&A, 355,891

Lelli, F., Verheijen, M., Fraternali, F., \& Sancisi, R. 2012, A\&A, 537, A72

Leroy, A., Bolatto, A. D., Simon, J. D., \& Blitz, L. 2005, ApJ, 625, 763

Leroy, A., Bolatto, A., Walter, F., \& Blitz, L. 2006, ApJ, 643, 825

Leroy, A., Cannon, J., Walter, F., Bolatto, A., \& Weiss, A. 2007, ApJ, 663, 990

Leroy, A. K., Walter, F., Bigiel, F., et al. 2009, AJ, 137, 4670

Leroy, A. K., Bolatto, A., Gordon, K., et al. 2011, ApJ, 737, 12

Lisenfeld, U., \& Ferrara, A. 1998, ApJ, 496, 145

Lisenfeld, U., Israel, F. P., Stil, J. M., \& Sievers, A. 2002, A\&A, 382, 860

López-Sánchez, Á. R., Koribalski, B. S., van Eymeren, J., et al. 2012, MNRAS, 419, 1051

Madden, S. C., Poglitsch, A., Geis, N., Stacey, G. J., \& Townes, C. H. 1997, ApJ, 483,200

Madden, S. C., Galliano, F., Jones, A. P., \& Sauvage, M. 2006, A\&A, 446, 877

Madden, S. C., Rémy, A., Galliano, F., et al. 2012, in IAU Symp. 284, eds. R. J. Tuffs, \& C. C. Popescu, 141

Madden, S. C., Rémy-Ruyer, A., Galametz, M., et al. 2013, PASP, 125, 600

Magdis, G. E., Daddi, E., Béthermin, M., et al. 2012, ApJ, 760, 6

Magnelli, B., Saintonge, A., Lutz, D., et al. 2012, A\&A, 548, A22

Magrini, L., Bianchi, S., Corbelli, E., et al. 2011, A\&A, 535, A13

Mathis, J. S., Mezger, P. G., \& Panagia, N. 1983, A\&A, 128, 212

McCall, M. L., Rybski, P. M., \& Shields, G. A. 1985, ApJS, 57, 1
McQuade, K., Calzetti, D., \& Kinney, A. L. 1995, ApJS, 97, 331 Meixner, M., Galliano, F., Hony, S., et al. 2010, A\&A, 518, L71 Meny, C., Gromov, V., Boudet, N., et al. 2007, A\&A, 468, 171

Meurer, G. R., Staveley-Smith, L., \& Killeen, N. E. B. 1998, MNRAS, 300, 705

Moles, M., Marquez, I., Masegosa, J., et al. 1994, ApJ, 432, 135

Montuori, M., Di Matteo, P., Lehnert, M. D., Combes, F., \& Semelin, B. 2010, A\&A, 518, A56

Moustakas, J., Kennicutt, Jr., R. C., Tremonti, C. A., et al. 2010, ApJS, 190, 233

Muñoz-Mateos, J. C., Gil de Paz, A., Boissier, S., et al. 2009, ApJ, 701, 1965

Neininger, N., Guelin, M., Garcia-Burillo, S., Zylka, R., \& Wielebinski, R. 1996, A\&A, 310, 725

O'Halloran, B., Galametz, M., Madden, S. C., et al. 2010, A\&A, 518, L58

Paturel, G., Theureau, G., Bottinelli, L., et al. 2003, A\&A, 412, 57

Pilleri, P., Montillaud, J., Berné, O., \& Joblin, C. 2012, A\&A, 542, A69

Pilyugin, L. S., \& Thuan, T. X. 2005, ApJ, 631, 231

Pilyugin, L. S., Thuan, T. X., \& Vílchez, J. M. 2006, MNRAS, 367, 1139

Poglitsch, A., Krabbe, A., Madden, S. C., et al. 1995, ApJ, 454, 293

Pustilnik, S. A., \& Martin, J.-M. 2007, A\&A, 464, 859

Pustilnik, S. A., Martin, J.-M., Huchtmeier, W. K., et al. 2002, A\&A, 389, 405

Rémy-Ruyer, A., Madden, S. C., Galliano, F., et al. 2013, A\&A, 557, A95

Revaz, Y., \& Jablonka, P. 2012, A\&A, 538, A82

Sage, L. J., Salzer, J. J., Loose, H.-H., \& Henkel, C. 1992, A\&A, 265, 19

Sandstrom, K. M., Leroy, A. K., Walter, F., et al. 2013, ApJ, 777, 5

Sauvage, M., Thuan, T. X., \& Lagage, P. O. 1997, A\&A, 325, 98

Savage, B. D., \& Sembach, K. R. 1996, ARA\&A, 34, 279

Schruba, A., Leroy, A. K., Walter, F., et al. 2012, AJ, 143, 138

Searle, L., \& Sargent, W. L. W. 1972, ApJ, 173, 25

Simpson, C. E., \& Gottesman, S. T. 2000, AJ, 120, 2975

Skibba, R. A., Engelbracht, C. W., Dale, D., et al. 2011, ApJ, 738, 89

Smith, M. W. L., Gomez, H. L., Eales, S. A., et al. 2012, ApJ, 748, 123

Strong, A. W., Moskalenko, I. V., Reimer, O., Digel, S., \& Diehl, R. 2004, A\&A, 422, L47

Swaters, R. A., van Albada, T. S., van der Hulst, J. M., \& Sancisi, R. 2002, A\&A, 390,829

Taylor, C. L., Kobulnicky, H. A., \& Skillman, E. D. 1998, AJ, 116, 2746

Thuan, T. X., \& Martin, G. E. 1981, ApJ, 247, 823

Thuan, T. X., Lipovetsky, V. A., Martin, J.-M., \& Pustilnik, S. A. 1999, A\&AS, 139,1

Thuan, T. X., Hibbard, J. E., \& Lévrier, F. 2004, AJ, 128, 617

Tolstoy, E., Hill, V., \& Tosi, M. 2009, ARA\&A, 47, 371

Valcke, S., de Rijcke, S., \& Dejonghe, H. 2008, MNRAS, 389, 1111

van Eymeren, J., Marcelin, M., Koribalski, B. S., et al. 2009, A\&A, 505, 105

van Zee, L., Westpfahl, D., Haynes, M. P., \& Salzer, J. J. 1998, AJ, 115, 1000

van Zee, L., Salzer, J. J., \& Skillman, E. D. 2001, AJ, 122, 121

Veilleux, S., Kim, D.-C., Sanders, D. B., Mazzarella, J. M., \& Soifer, B. T. 1995, ApJS, 98, 171

Walter, F., Taylor, C. L., Hüttemeister, S., Scoville, N., \& McIntyre, V. 2001, AJ, 121,727

Walter, F., Brinks, E., de Blok, W. J. G., et al. 2008, AJ, 136, 2563

Whittet, D. C. B. 2003, Astron. Geophys., 44, 35

Williams, B. A., McMahon, P. M., \& van Gorkom, J. H. 1991, AJ, 101, 1957

Wilson, C. D. 1995, ApJ, 448, L97

Wilson, C. D., Warren, B. E., Israel, F. P., et al. 2012, MNRAS, 424, 3050

Young, J. S., \& Knezek, P. M. 1989, ApJ, 347, L55

Young, J. S., Xie, S., Tacconi, L., et al. 1995, ApJS, 98, 219

Young, L. M., Bureau, M., Davis, T. A., et al. 2011, MNRAS, 414, 940

Ysard, N., \& Verstraete, L. 2010, A\&A, 509, A12

Zhu, M., Papadopoulos, P. P., Xilouris, E. M., Kuno, N., \& Lisenfeld, U. 2009, ApJ, 706, 941

Zhukovska, S. 2008, Ph.D. thesis, Ruperto-Carola-University

Zhukovska, S. 2014, A\&A, 562, A76

Zhukovska, S., Gail, H.-P., \& Trieloff, M. 2008, A\&A, 479, 453

Zubko, V., Dwek, E., \& Arendt, R. G. 2004, ApJS, 152, 211 


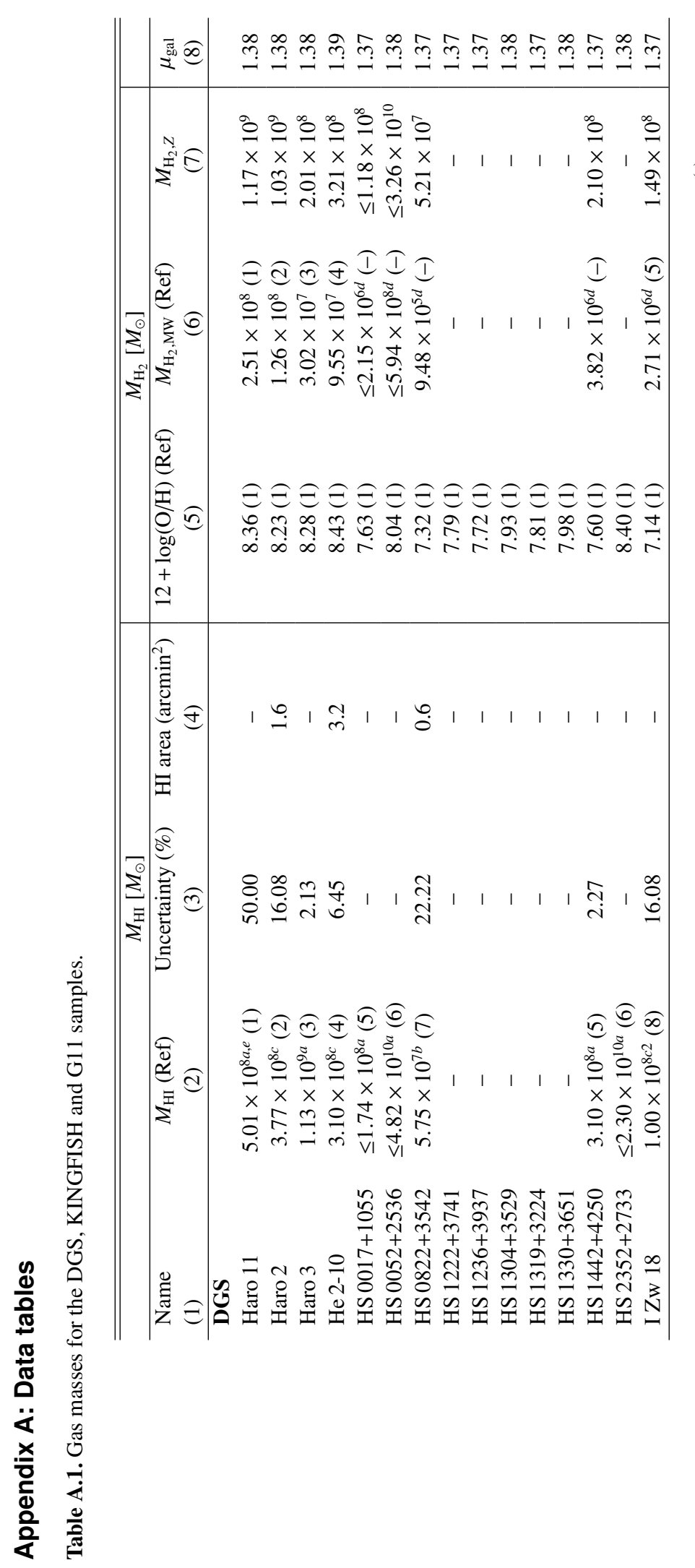

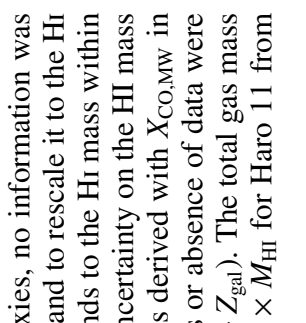

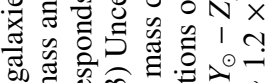

on

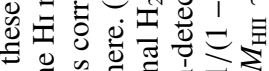

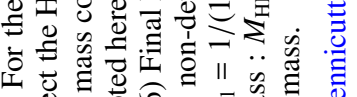

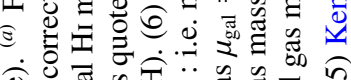

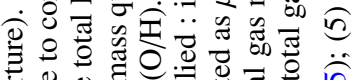

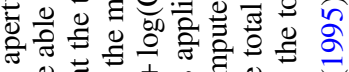

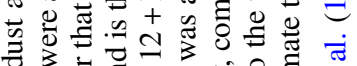

o

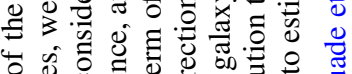

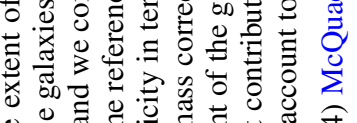

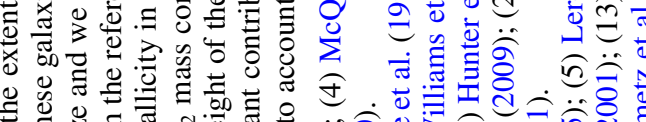

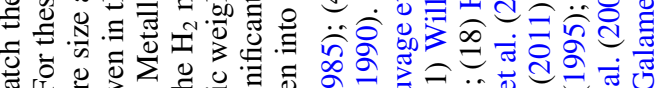

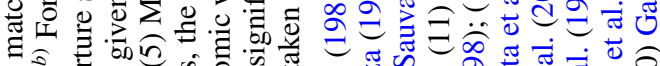

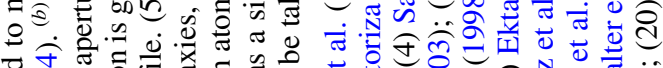

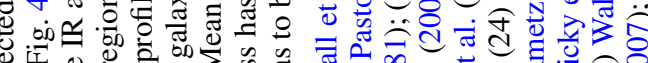

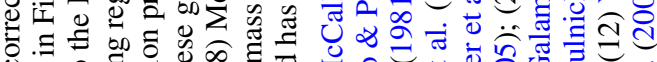

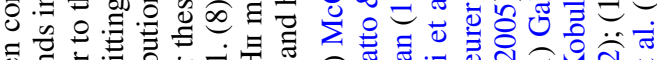

ฮ D.:

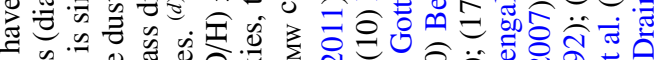

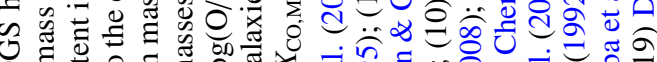

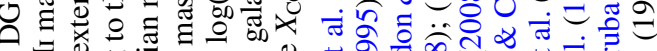

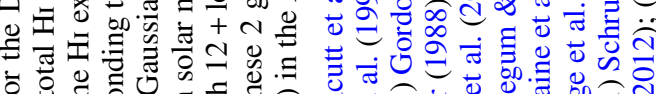
의의.

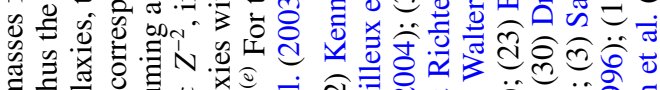

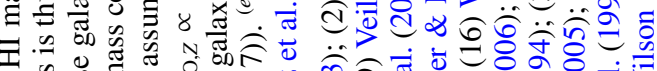

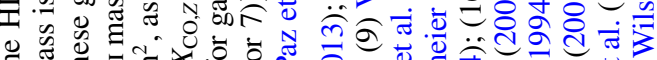

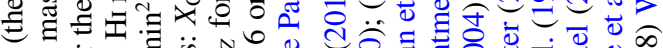

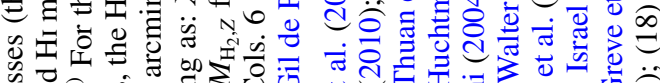

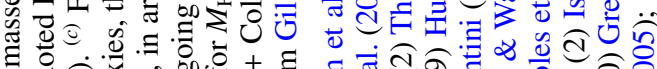

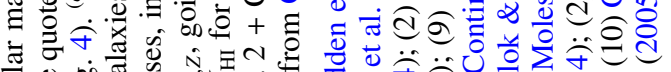

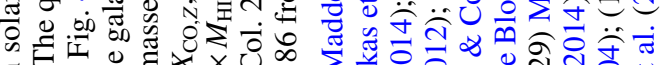

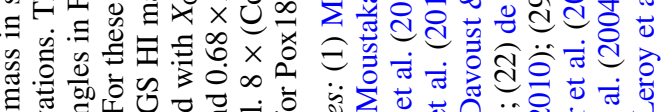

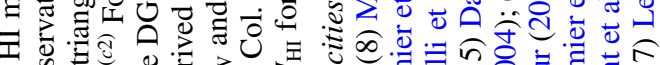

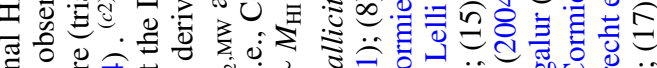

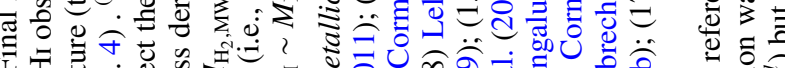

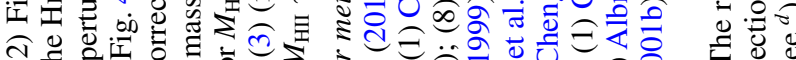
ن

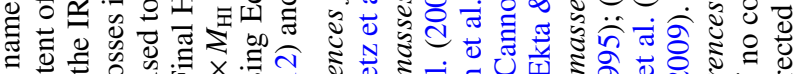

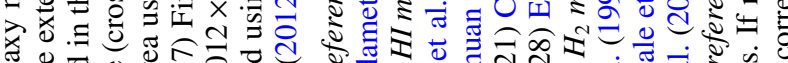

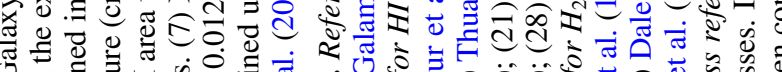

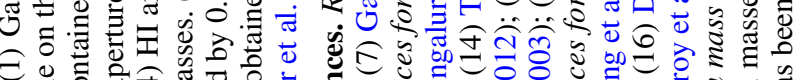

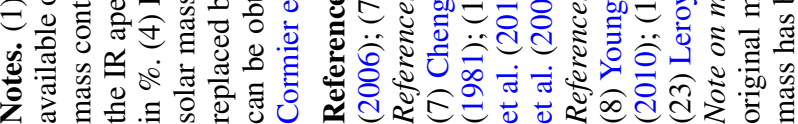




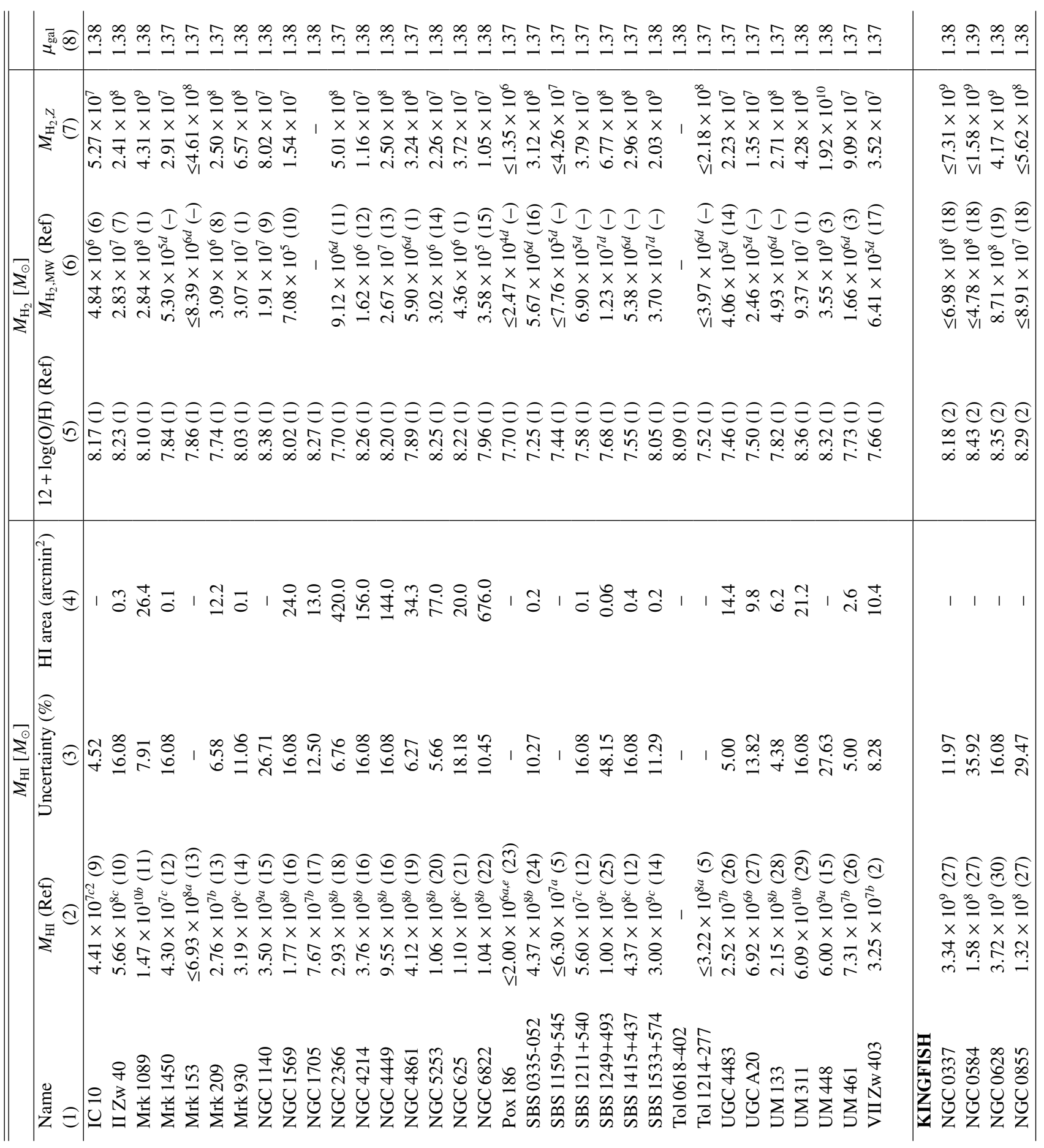




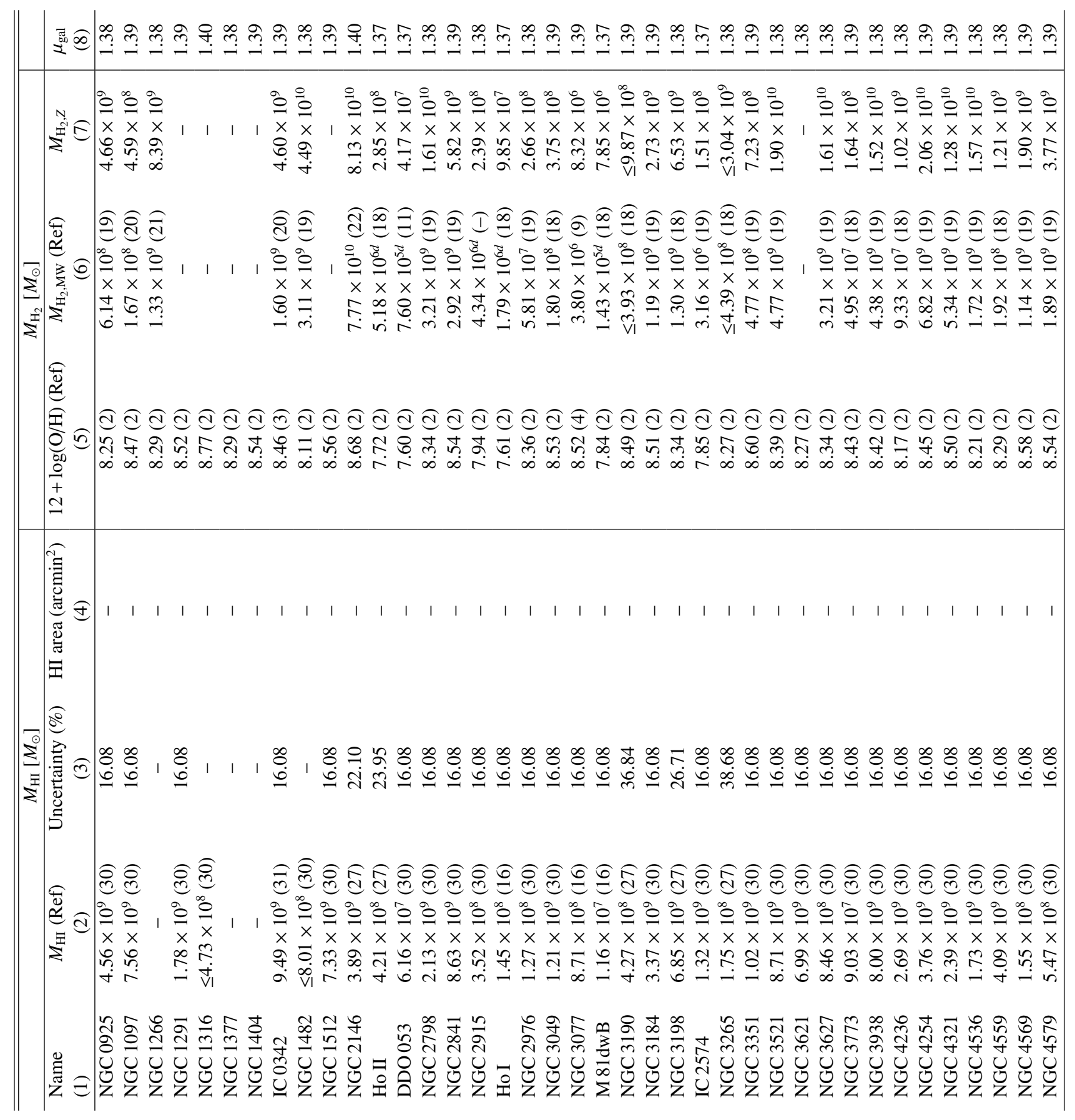




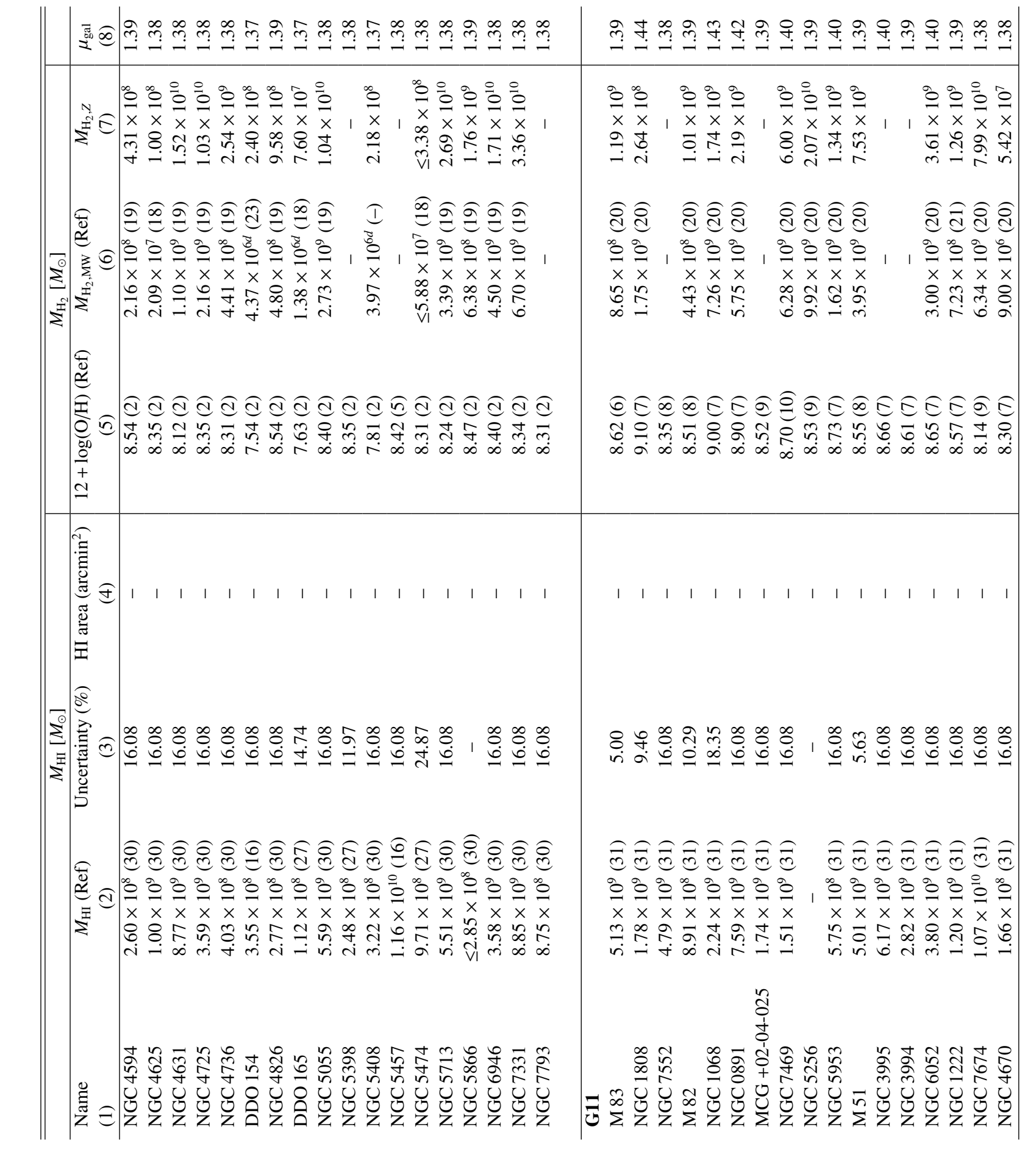


Table A.2. Dust masses for the KINGFISH and G11 samples.

\begin{tabular}{|c|c|c|}
\hline Name & $M_{\text {dust }}\left[M_{\odot}\right]$ & Uncertainty (\%) \\
\hline \multicolumn{3}{|l|}{ KINGFISH } \\
\hline NGC 0337 & $1.60 \times 10^{7}$ & 8.14 \\
\hline NGC 0584 & - & - \\
\hline NGC 0628 & $2.57 \times 10^{7}$ & 12.42 \\
\hline NGC 0855 & $1.84 \times 10^{6}$ & 22.90 \\
\hline NGC 0925 & $2.52 \times 10^{7}$ & 14.44 \\
\hline NGC 1097 & $8.29 \times 10^{7}$ & 8.05 \\
\hline NGC 1266 & $8.43 \times 10^{6}$ & 7.27 \\
\hline NGC 1291 & $1.64 \times 10^{7}$ & 15.67 \\
\hline NGC 1316 & $9.35 \times 10^{6}$ & 10.99 \\
\hline NGC 1377 & $1.47 \times 10^{6}$ & 10.67 \\
\hline NGC 1404 & - & - \\
\hline IC 0342 & $3.95 \times 10^{7}$ & 7.34 \\
\hline NGC 1482 & $2.54 \times 10^{7}$ & 7.91 \\
\hline NGC 1512 & $3.09 \times 10^{7}$ & 27.99 \\
\hline NGC 2146 & $4.75 \times 10^{7}$ & 8.23 \\
\hline Ho II & $7.58 \times 10^{4}$ & 7.90 \\
\hline DDO 053 & $1.04 \times 10^{4}$ & 13.38 \\
\hline NGC 2798 & $1.24 \times 10^{7}$ & 6.86 \\
\hline NGC 2841 & $5.91 \times 10^{7}$ & 9.11 \\
\hline NGC 2915 & $3.38 \times 10^{5}$ & 21.07 \\
\hline Ho I & $2.38 \times 10^{5}$ & 8.27 \\
\hline NGC 2976 & $1.89 \times 10^{6}$ & 9.07 \\
\hline NGC 3049 & $7.69 \times 10^{6}$ & 20.60 \\
\hline NGC 3077 & $1.13 \times 10^{6}$ & 15.35 \\
\hline M 81dwB & $2.81 \times 10^{4}$ & 63.98 \\
\hline NGC 3190 & $1.82 \times 10^{7}$ & 11.09 \\
\hline NGC 3184 & $3.16 \times 10^{7}$ & 12.82 \\
\hline NGC 3198 & $3.76 \times 10^{7}$ & 13.32 \\
\hline IC 2574 & $2.79 \times 10^{6}$ & 47.44 \\
\hline NGC 3265 & $1.84 \times 10^{6}$ & 11.68 \\
\hline NGC 3351 & $1.93 \times 10^{7}$ & 6.82 \\
\hline NGC 3521 & $7.08 \times 10^{7}$ & 12.08 \\
\hline NGC 3621 & $1.89 \times 10^{7}$ & 12.70 \\
\hline NGC 3627 & $4.04 \times 10^{7}$ & 7.52 \\
\hline NGC 3773 & $7.56 \times 10^{5}$ & 12.46 \\
\hline NGC 3938 & $4.65 \times 10^{7}$ & 13.22 \\
\hline NGC 4236 & $2.67 \times 10^{6}$ & 14.51 \\
\hline NGC 4254 & $5.83 \times 10^{7}$ & 9.45 \\
\hline NGC 4321 & $7.23 \times 10^{7}$ & 11.64 \\
\hline NGC 4536 & $3.11 \times 10^{7}$ & 11.81 \\
\hline NGC 4559 & $9.56 \times 10^{6}$ & 11.95 \\
\hline NGC 4569 & $1.58 \times 10^{7}$ & 6.72 \\
\hline NGC 4579 & $3.30 \times 10^{7}$ & 5.98 \\
\hline NGC 4594 & $2.06 \times 10^{7}$ & 17.07 \\
\hline NGC 4625 & $1.99 \times 10^{6}$ & 14.87 \\
\hline NGC 4631 & $3.48 \times 10^{7}$ & 9.40 \\
\hline NGC 4725 & $4.45 \times 10^{7}$ & 9.86 \\
\hline NGC 4736 & $5.64 \times 10^{6}$ & 11.28 \\
\hline DDO 154 & - & - \\
\hline NGC 4826 & $4.31 \times 10^{6}$ & 8.08 \\
\hline
\end{tabular}

Table A.2. continued.

\begin{tabular}{lcc}
\hline \hline Name & $M_{\text {dust }}\left[M_{\odot}\right]$ & Uncertainty $(\%)$ \\
\hline DDO 165 & - & - \\
NGC 5055 & $5.39 \times 10^{7}$ & 8.21 \\
NGC 5398 & $3.06 \times 10^{6}$ & 43.26 \\
NGC 5408 & $8.50 \times 10^{4}$ & 25.68 \\
NGC 5457 & $6.62 \times 10^{7}$ & 15.15 \\
NGC 5474 & $3.73 \times 10^{6}$ & 12.30 \\
NGC 5713 & $2.78 \times 10^{7}$ & 7.68 \\
NGC 5866 & $6.05 \times 10^{6}$ & 9.06 \\
NGC 6946 & $7.27 \times 10^{7}$ & 6.24 \\
NGC 7331 & $1.05 \times 10^{8}$ & 8.57 \\
NGC 7793 & $6.41 \times 10^{6}$ & 13.41 \\
& & \\
\hline G11 & & \\
M 83 & $1.10 \times 10^{7}$ & 8.14 \\
NGC 1808 & $2.54 \times 10^{7}$ & 34.24 \\
NGC 7552 & $7.24 \times 10^{7}$ & 16.10 \\
M 82 & $6.85 \times 10^{6}$ & 33.02 \\
NGC 1068 & $2.77 \times 10^{8}$ & 36.05 \\
NGC 0891 & $5.84 \times 10^{7}$ & 11.84 \\
MGC +02-04-025 & $1.01 \times 10^{8}$ & 47.08 \\
NGC 7469 & $1.55 \times 10^{8}$ & 19.16 \\
NGC 5256 & $2.01 \times 10^{8}$ & 62.93 \\
NGC 5953 & $2.25 \times 10^{7}$ & 18.90 \\
M 51 & $3.74 \times 10^{8}$ & 21.82 \\
NGC 3995 & $5.75 \times 10^{7}$ & 23.47 \\
NGC 3994 & $5.27 \times 10^{7}$ & 34.81 \\
NGC 6052 & $5.84 \times 10^{7}$ & 32.58 \\
NGC 1222 & $1.33 \times 10^{7}$ & 26.71 \\
NGC 7674 & $9.83 \times 10^{7}$ & 29.69 \\
NGC 4670 & $1.13 \times 10^{6}$ & 21.12 \\
\hline & & \\
\hline
\end{tabular}

Notes. The dust masses for the DGS sample are given in Rémy-Ruyer et al., in prep. 
A. Rémy-Ruyer et al.: Gas-to-dust mass ratios in local galaxies over a 2 dex metallicity range

\section{Appendix B: G/D with morphological type, stellar mass and star formation rate}

In this appendix, we present how the sample distributes in morphological types, stellar masses and star formation rates (Fig. B.2). The morphological types were taken from NED. The stellar masses were obtained from the formula of Eskew et al. (2012) using the 3.6 and $4.5 \mu \mathrm{m}$ IRAC flux densities. Whenever IRAC data was not available, we compute synthetic IRAC photometry from the SEDs. The SFR were converted from TIR luminosities using the Kennicutt (1998) formula. We also present the G/D as a function of these three parameters (Fig. B.3) and as a function of the specific star formation rate (SSFR, Fig. B.1). SSFR is defined as the star formation rate divided by the stellar mass: $S F R / M_{*}$.
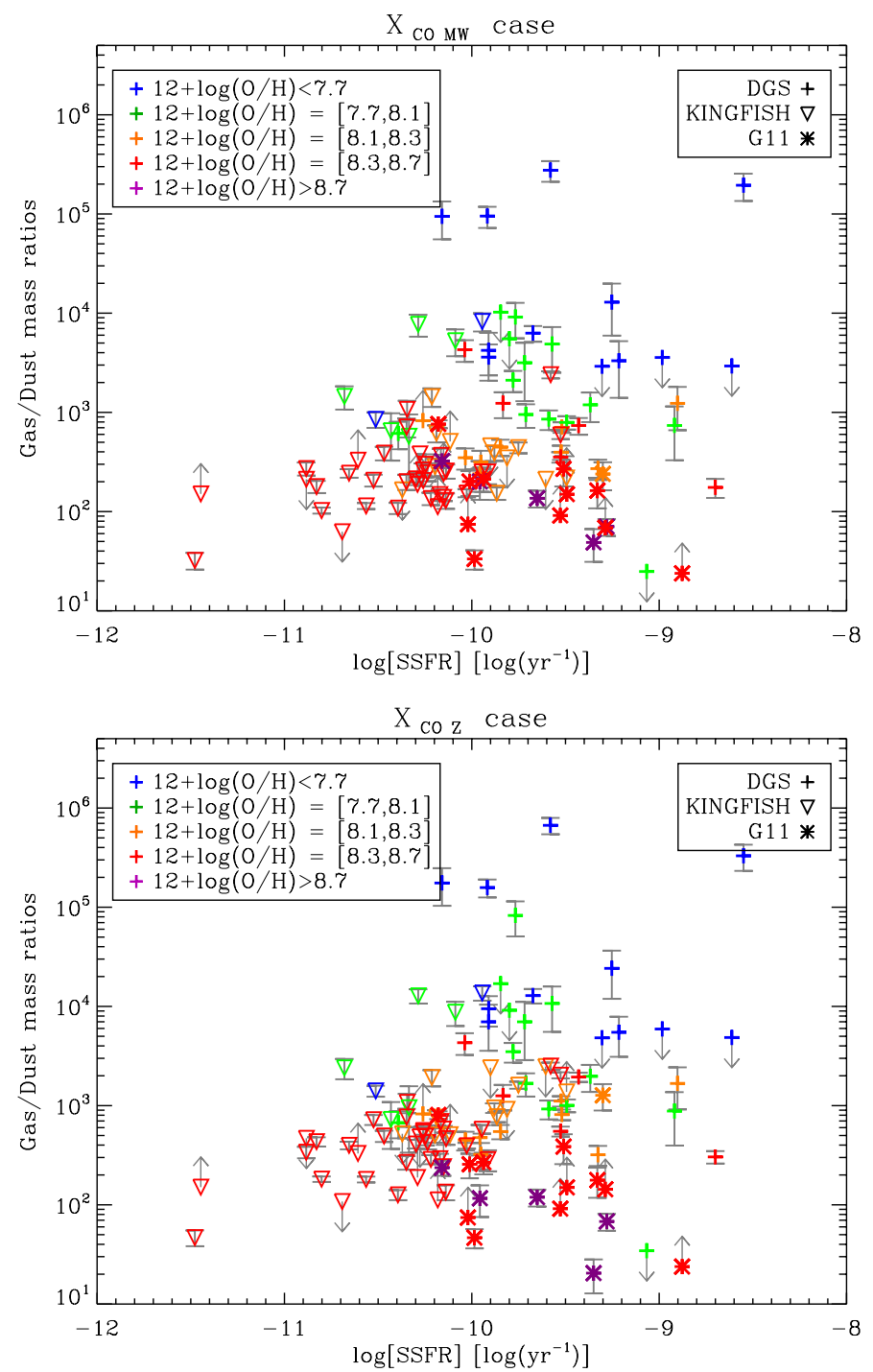

Fig. B.1. G/D as a function of SSFR for the 2 values of $X_{\mathrm{CO}}: X_{\mathrm{CO}, \mathrm{MW}}$ (top) and $X_{\mathrm{CO}, Z}$ (bottom). The colours code for the metallicity of the galaxies and the symbols differentiate between the three samples: DGS (crosses), KINGFISH (downward triangles) and G11 (stars).
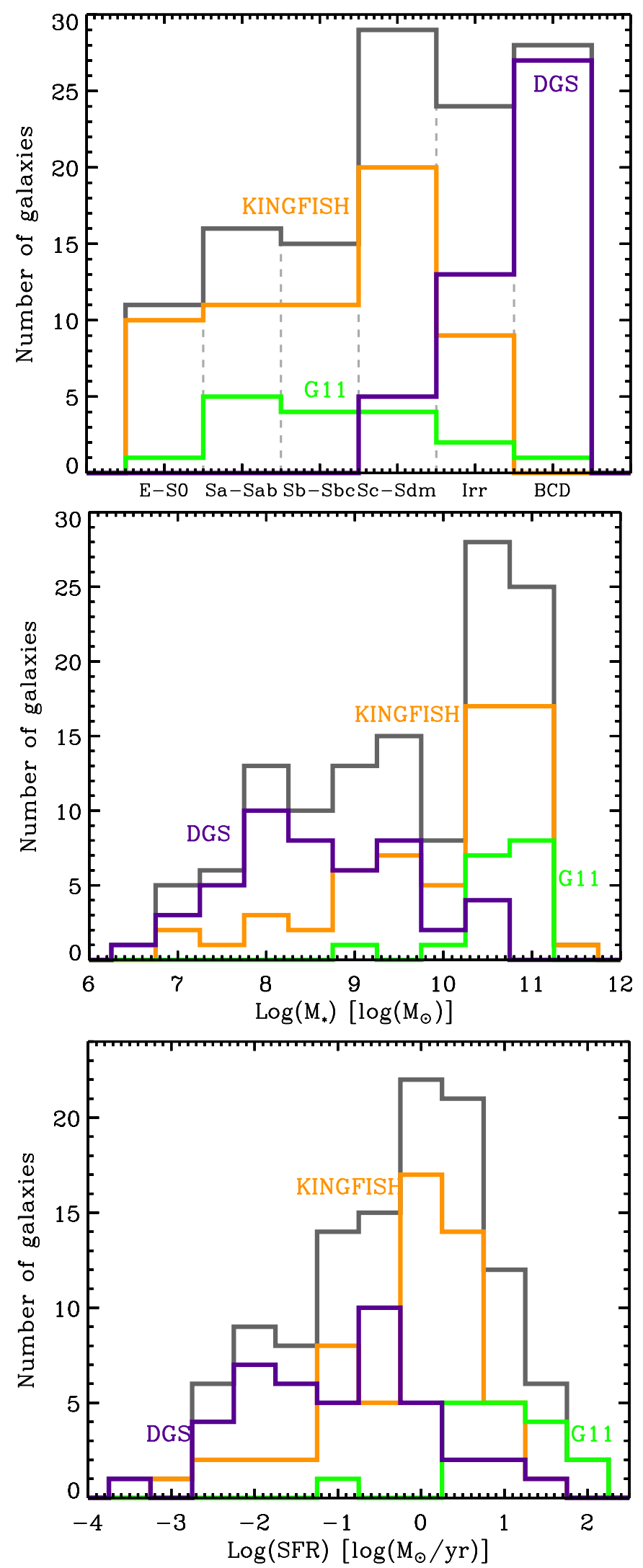

Fig. B.2. Morphological type distributions (top), stellar mass distributions (centre), and star formation rate distributions (bottom) of the DGS (purple), KINGFISH (orange) and G11 (green) samples. In each panel, the total distribution is indicated in grey. 

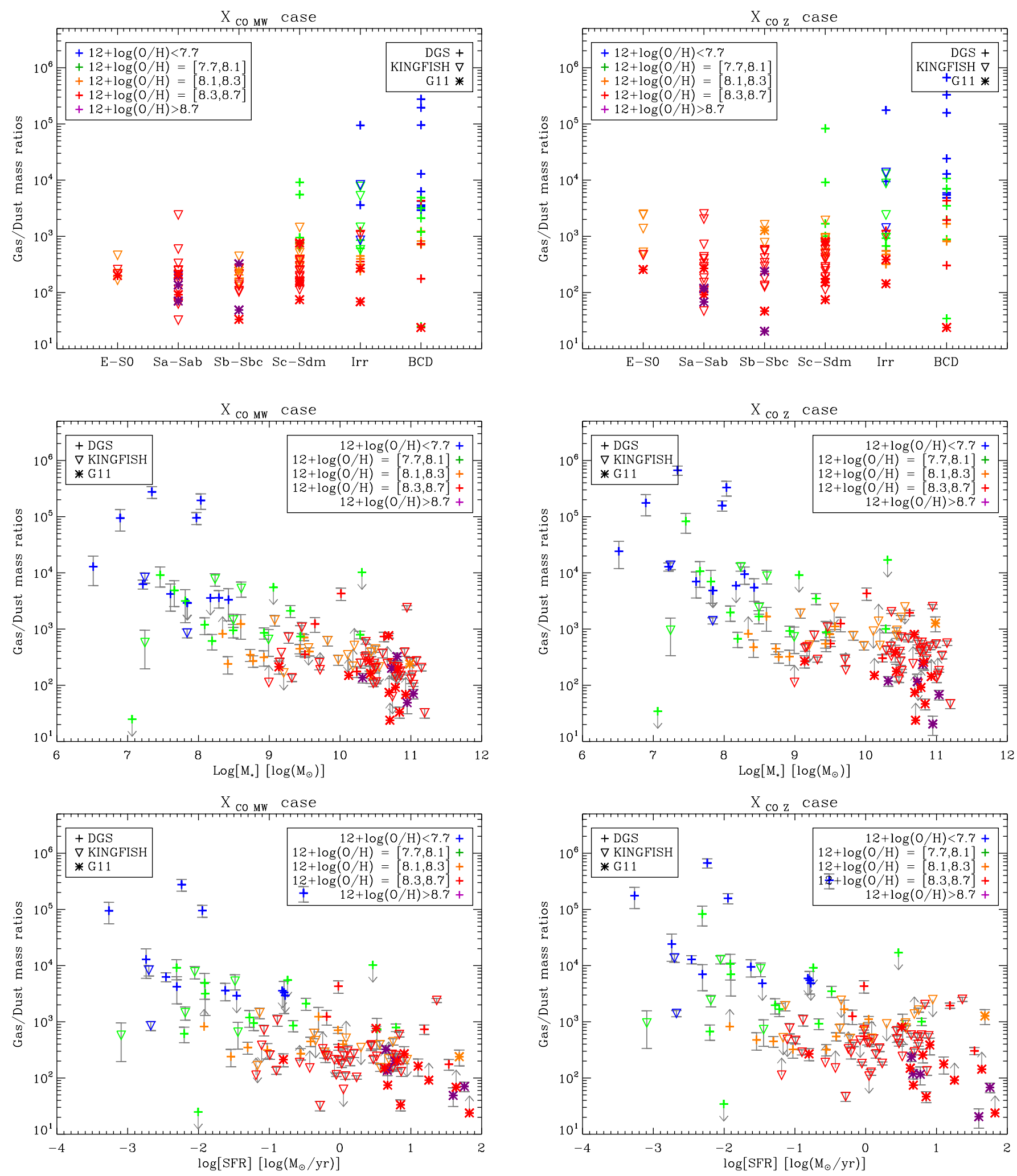

Fig. B.3. G/D as a function of morphological type (top row), stellar mass (centre row) and star formation rate (bottom row) for the 2 values of $X_{\mathrm{CO}}$ : $X_{\mathrm{CO}, \mathrm{Mw}}$ (left column) and $X_{\mathrm{CO}, Z}$ (right column). The colours code for the metallicity of the galaxies and the symbols differentiate between the three samples: DGS (crosses), KINGFISH (downward triangles) and G11 (stars). 